\title{
Two classes of quasi-steady-state model reductions for stochastic kinetics
}

\author{
Ethan A. Mastny ${ }^{\text {a) }}$ \\ Department of Chemical and Biological Engineering, University of Wisconsin-Madison, Madison, \\ Wisconsin 53706-1607, USA \\ Eric L. Haseltine ${ }^{\text {b) }}$ \\ Division of Chemistry and Chemical Engineering 210-41, California Institute of Technology, Pasadena, \\ California 91125, USA
}

\author{
James B. Rawlings ${ }^{\text {C) }}$ \\ Department of Chemical and Biological Engineering, University of Wisconsin-Madison, Madison, \\ Wisconsin 53706-1607, USA
}

(Received 7 February 2007; accepted 2 July 2007; published online 6 September 2007)

\begin{abstract}
The quasi-steady-state approximation (QSSA) is a model reduction technique used to remove highly reactive species from deterministic models of reaction mechanisms. In many reaction networks the highly reactive intermediates (QSSA species) have populations small enough to require a stochastic representation. In this work we apply singular perturbation analysis to remove the QSSA species from the chemical master equation for two classes of problems. The first class occurs in reaction networks where all the species have small populations and the QSSA species sample zero the majority of the time. The perturbation analysis provides a reduced master equation in which the highly reactive species can sample only zero, and are effectively removed from the model. The reduced master equation can be sampled with the Gillespie algorithm. This first stochastic QSSA reduction is applied to several example reaction mechanisms (including Michaelis-Menten kinetics) [Biochem. Z. 49, 333 (1913)]. A general framework for applying the first QSSA reduction technique to new reaction mechanisms is derived. The second class of QSSA model reductions is derived for reaction networks where non-QSSA species have large populations and QSSA species numbers are small and stochastic. We derive this second QSSA reduction from a combination of singular perturbation analysis and the $\Omega$ expansion. In some cases the reduced mechanisms and reaction rates from these two stochastic QSSA models and the classical deterministic QSSA reduction are equivalent; however, this is not usually the case. () 2007 American Institute of Physics. [DOI: $10.1063 / 1.2764480]$
\end{abstract}

\section{INTRODUCTION}

Time scales that span many orders of magnitude arise in reactive networks ranging from virus infection dynamics, to heterogeneous catalysis, and combustion. Classically these systems are modeled with ordinary differential equations and the fast time scales are removed with approximation techniques. When the numbers of reactive molecules are small, such as in a cell or on a catalyst particle, the intrinsic noise or spatial inhomogeneity necessitates a stochastic representation of the system. Multiple time scales often arise in systems where the stochastic representation is important.

When particle numbers are small and a stochastic representation of the system is necessary, the system can be modeled as a discrete Markov process where jumps from one discrete state to another represent chemical reactions. The master equation describes the time evolution of the probability of visiting any state. The stochastic simulation algorithm (SSA), originally proposed by Gillespie, ${ }^{1,2}$ advances a simulation by executing single reaction events. Simulating SSA is

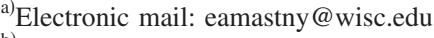

${ }^{b)}$ Electronic mail: haseltin@ caltech.edu

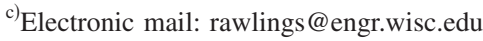

equivalent to sampling the probability distribution specified in the chemical master equation. The time step and probability of execution of each reaction are determined by the reaction rates.

In stochastic simulations multiple time scales can arise in at least three different ways: ${ }^{3}$

(1) Some set of reversible reactions occur frequently, and the remaining reactions occur rarely. This situation is analogous to reaction equilibrium.

(2) Some set of irreversible reactions occur frequently, and the remaining reactions occur rarely. Generally for this situation to be sustained some species numbers have to be large.

(3) Some species react so rapidly that their average number throughout the simulations is nearly zero or their average number is much smaller than the other species numbers.

In the last few years several works have been devoted to develop reduced master equations and efficient sampling schemes for reaction mechanisms with the first phenomenon of rapidly equilibrating reactions, ${ }^{3-7}$ and with the second phenomenon of many fast irreversible reactions. ${ }^{8-13}$ In this 
work, we are interested in addressing the third phenomenon. In particular, we seek to derive reduced chemical kinetics models that remove highly reactive intermediates. In this paper, we call the class of model reductions used to eliminate highly reactive species quasi-steady-state approximation (QSSA) methods, and the eliminated species are called QSSA species. When referring to QSSA we are not specifying a specific reduction procedure. In the paper we use other labels to specify procedures that are used to make QSSA model reductions.

\section{A. Deterministic QSSA reductions}

The following set of ordinary differential equations are often used to model chemical reaction networks in the deterministic limit, where $x$ and $y$ are concentration vectors of the non-QSSA and QSSA species, respectively.

$$
\frac{d x}{d t}=f(x, y), \quad \epsilon \frac{d y}{d t}=g(x, y) .
$$

The classical method for performing a QSSA reduction on Eq. (1) is to set the production rates of the QSSA species to zero, and solve the resulting set of differential algebraic equations (DAES). ${ }^{14,15}$

$$
\frac{d x}{d t}=f(x, y), \quad 0=g(x, y) .
$$

This set of DAES often has fewer kinetic parameters than the original model. If the algebraic equation can be solved explicitly for $y$ in terms of $x$, then $y(x)$ can be substituted into the differential equation and eliminate the algebraic equation from the model. In this paper we call the above approach deterministic QSSA classical (dQC).

Another methodology used to remove highly reactive intermediates from deterministic models is singular perturbation analysis (SPA). Perturbation methods represent solutions as a power series in a small parameter $(\epsilon)$.

$$
\begin{aligned}
& x=X_{0}+\epsilon X_{1}+\epsilon^{2} X_{2}+\mathcal{O}\left(\epsilon^{3}\right), \\
& y=Y_{0}+\epsilon Y_{1}+\epsilon^{2} Y_{2}+\mathcal{O}\left(\epsilon^{3}\right) .
\end{aligned}
$$

Equation (1) will result in a singular perturbation because the highest-order derivative is multiplied by $\epsilon$. In the case of singular perturbations a solution cannot be found for all values of the independent variable, and different approximations need to be found for each region of the independent variable. In the above example different power series approximation are needed for the fast and slow time scales. ${ }^{16}$ The fast and slow time-scale solutions are often called the inner and outer solutions, respectively. In this paper we will call the above approach deterministic QSSA SPA (dQSPA).

The solutions to both the dQC and dQSPA converge to the solution of the original model as $\epsilon$ goes to zero; ${ }^{17}$ however, for finite $\epsilon$ the order of the error is known for dQSPA while it is not known for dQC. Additionally for finite $\epsilon$ the nonlinear algebraic equations in dQC may not have closed form solutions for $y$ at a given $x$. Another advantage of dQSPA over dQC is that by investigating each order of the power series separately additional insight can be gained on the behavior of the system, while the dQC solution is a mixture of all the orders.

\section{B. Stochastic QSSA reductions}

Here, we seek to address highly reactive species in the stochastic setting. We impose two requirements for our reduced models: (i) the reduced models needs to be simpler than the full model (fewer reactions, fewer parameters to be identified from data, or faster simulation times, for example), and (ii) the solutions of the reduced models need to converge to the full model as a specified parameter goes to zero. Reduced kinetic models that meet our two requirements are not unique, for example, both $\mathrm{dQC}$ and the zero-order outer dQSPA satisfy these requirements for deterministic systems.

The master equation for well mixed chemical reaction networks is

$$
\begin{aligned}
\frac{d P\left(n_{s}, n_{f}\right)}{d t}= & \sum_{j=1}^{N_{r x n}} k_{j} a_{j}\left(n_{s}-\nu_{j}^{s}, n_{f}-\nu_{j}^{f}\right) P\left(n_{s}-\nu_{j}^{s}, n_{f}-\nu_{j}^{f}\right) \\
& -k_{j} a_{j}\left(n_{s}, n_{f}\right) P\left(n_{s}, n_{f}\right),
\end{aligned}
$$

in which $n_{s}$ and $n_{f}$ are vectors of integers representing nonQSSA and QSSA species numbers, $P\left(n_{s}, n_{f}\right)$ is the probability of being in state $\left(n_{s}, n_{f}\right), k_{j}$ is the rate constant of reaction $j, k_{j} a_{j}\left(n_{s}, n_{f}\right)$ is the rate of reaction $j$, and $\nu_{j}^{s}$ and $\nu_{j}^{f}$ are the columns of the non-QSSA and QSSA stoichiometric matrices that corresponds to reaction $j$.

An analog to the classical deterministic approach (dQC) in the stochastic setting is to find a set of DAES that give the time evolution of the non-QSSA species probability density $\left(P\left(n_{s}\right)\right)$. The evolution equation for $P\left(n_{s}\right)$ is

$$
\begin{aligned}
\frac{d P\left(n_{s}\right)}{d t}= & \sum_{n_{f}} \sum_{j=1}^{N_{r x n}} k_{j} a_{j}\left(n_{s}-\nu_{j}^{s}, n_{f}-\nu_{j}^{f}\right) P\left(n_{f}-\nu_{j}^{f} \mid n_{s}-\nu_{j}^{s}\right) \\
& \times P\left(n_{s}-\nu_{j}^{s}\right)-k_{j} a_{j}\left(n_{s}, n_{f}\right) P\left(n_{f} \mid n_{s}\right) P\left(n_{s}\right),
\end{aligned}
$$

where $P\left(n_{f} \mid n_{s}\right)$ is the conditional probability of being in state $n_{f}$ given state $n_{s}$.

The algebraic equations come from setting the rate of change of the conditional probability of the QSSA states on the non-QSSA states to zero.

$$
\frac{d P\left(n_{f} \mid n_{s}\right)}{d t}=0 .
$$

Applying the definition of conditional probability to the master equation gives the following algebraic constraint:

$$
0=\left(\frac{d P\left(n_{s}, n_{f}\right)}{d t}-P\left(n_{f} \mid n_{s}\right) \frac{d P\left(n_{s}\right)}{d t}\right) \frac{1}{P\left(n_{s}\right)} .
$$

For a given $P\left(n_{s}\right)$, Eq. (6) could possibly be solved for $P\left(n_{f} \mid n_{s}\right)$, although this is a large set of nonlinear equations. Equation (6) coupled to Eq. (5) gives a reduced model analogous to dQC.

Rao and Arkin ${ }^{18}$ proposed to use this classical reduction procedure to apply QSSA to the master equation; however, to 
TABLE I. Description of the reduced QSSA models discussed in this paper.

\begin{tabular}{ll}
\hline \hline Model name & Description \\
\hline dQC & Deterministic QSSA, classical \\
dQSPA & Deterministic QSSA, singular perturbation analysis \\
SQSPA & Stochastic QSSA, singular perturbation analysis \\
sQSPA- $\Omega$ & $\begin{array}{l}\text { Stochastic QSSA, singular perturbation analysis, } \Omega \\
\text { expansion }\end{array}$ \\
\hline \hline
\end{tabular}

simplify the algebraic constraint they made an additional assumption that $P\left(n_{f} \mid n_{s}\right)$ evolves according to the following equation:

$$
\begin{aligned}
\frac{d P\left(n_{f} \mid n_{s}\right)}{d t} \approx & \sum_{j=1}^{N_{r x n}} k_{j} a_{j}\left(n_{s}-\nu_{j}^{s}, n_{f}-v_{j}^{f}\right) P\left(n_{f}-\nu_{j}^{f} \mid n_{s}\right) \\
& -k_{j} a_{j}\left(n_{s}, n_{f}\right) P\left(n_{f} \mid n_{s}\right) .
\end{aligned}
$$

Given this additional assumption and the ad hoc treatment of the non-QSSA stoichiometric coefficients $\left(\nu_{j}^{S}\right)$ in Eq. (7) it is unclear that their proposed reduced model converges to the full model solution in the limit of a parameter approaching zero. $^{5}$

Due to the difficultly of solving Eq. (6) we have chosen not to pursue this classical reduction technique. Instead we have chosen to apply SPA to derive reduced QSSA models from the master equation.

If a closed system has reactive species with small populations, and the reactions that consume the QSSA species are much faster than the other reactions, then the QSSA species numbers evolve rapidly to hit the value zero. Any reaction that produces a QSSA species is quickly followed by a reaction that consumes that species, therefore the time averaged population of the QSSA species is nearly zero. In this paper, we derive stochastic reduced models that do approach the full model when QSSA species numbers are nearly always zero. These reduced kinetic models are derived from the zero-order outer term of SPA applied to the master equation. We call this class of reduced models stochastic QSSA SPA (sQSPA).

If we increase the size of this system $(\Omega)$ in such a way that the concentrations remain constant, the QSSA species numbers remain nearly zero until the system size becomes sufficiently large. In the limit of the consumption rate parameter approaching infinity $(\epsilon \rightarrow 0)$, the highly reactive species numbers remain zero for a fixed $\Omega$. In the limit of $1 / \Omega \rightarrow 0$ and fixed $\epsilon$, the QSSA species numbers are nonzero and evolve deterministically. Situations may arise in which both $1 / \Omega$ and $\epsilon$ approach zero, and the resulting reduced model depends on which parameter approaches zero faster. In the case that $1 / \Omega$ and $\epsilon$ are both arbitrarily small but the product $\Omega \epsilon$ is a constant of $\mathcal{O}(1)$, the non-QSSA species are large and evolve deterministically, and the QSSA species numbers are small (though not necessarily zero) and require a stochastic description. In this paper, we apply SPA in conjunction with an $\Omega$ expansion to the master equation to develop reduced models for systems in this balanced state. We call this class of reduced models stochastic QSSA SPA- $\Omega$-expansion $(\mathrm{sQSPA}-\Omega)$.

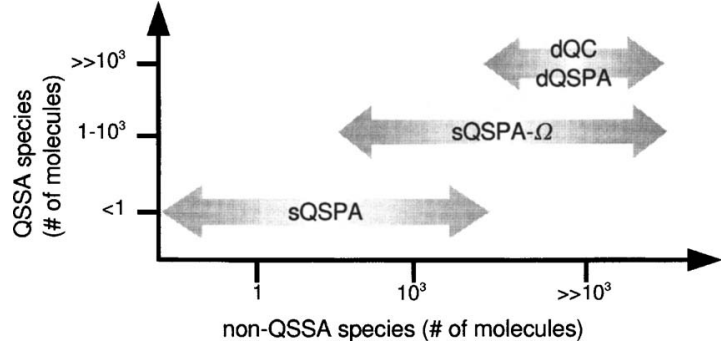

FIG. 1. Graphical description of the regions of applicability of the various QSSA models described in Table I.

Other works have sought to develop QSSA reduced models in the stochastic setting. Janssen ${ }^{19,20}$ used the projection operator method ${ }^{21}$ to try eliminate highly reactive species from the chemical master equation. In some cases the method yields a reduced master equation. However, other reaction mechanisms are irreducible with the projection operator method. Reduced master equations derived using projection operators track the populations of transformed variables rather than the non-QSSA species. The reduced master equations are different than those derived by the SPA proposed here, and do not approach the full model as specific parameters become zero. Additionally, we have developed a reduced model using stochastic QSSA in Sec. VI B for a mechanism that Janssen called irreducible with the projection operator method. ${ }^{20}$

Recently Peles et al. proposed the use of SPA to develop reduced master equations for chemical kinetics when multiple time scales are present. ${ }^{22}$ Their use of SPA identifies clusters of states within which transitions occur rapidly, while transitions between the clusters are rare. Their reduced master equation tracks the transitions from one cluster to another. However, calculating the transition rates between clusters requires the solution of a nontrivial eigenvector problem. While their method is well suited for reaction equilibrium problems, we believe that it is overly complex for QSSA problems.

Several different methods for creating QSSA reduced models are discussed in this paper. Table I summarizes the names we use to describe these models throughout the paper. Figure 1 shows a graphical description of the approximate population numbers of the QSSA and non-QSSA species that are appropriate for the models given in Table I.

This paper is organized as follows. In Secs. II-VI the master equation order reduction where system size is small (sQSPA) is presented. In Sec. II, an illustration of singular perturbation applied to the master equation in the QSSA limit is given. In Sec. III, a table with several examples of mechanisms that have been reduced with SQSPA is presented. Then in Sec. IV, a general derivation of sQSPA from singular perturbation analysis is given along with discussion. In Sec. V, a comparison of sQSPA and dQC models for enzyme kinetics is presented. In Sec. VI, methods for treating two cases that are not addressed in the general derivation of SQSPA are presented. Section VII discusses the application of SPA and the $\Omega$ expansion to the master equation (sQSPA- $\Omega$ ). 


\section{SQSPA EXAMPLE}

To conceptually illustrate SQSPA, we consider the following elementary mechanism:

$$
\begin{aligned}
& \underset{k_{-1}}{\stackrel{k_{1}}{\rightleftharpoons} 2 \mathrm{~B}}, \quad r_{1}=k_{1} a, \quad r_{-1}=\frac{1}{2} k_{-1} b(b-1) \\
& B \stackrel{k_{2}}{\longrightarrow} \mathrm{C}, \quad r_{2}=k_{2} b .
\end{aligned}
$$

This mechanism is governed by the following master equation:

$$
\begin{aligned}
\frac{d P(a, b, c)}{d t}= & k_{1}(a+1) P(a+1, b-2, c) \\
& +\frac{1}{2} k_{-1}(b+2)(b+1) P(a-1, b+2, c) \\
& +k_{2}(b+1) P(a, b+1, c-1) \\
& -\left(k_{1} a+\frac{1}{2} k_{-1} b(b-1)+k_{2} b\right) P(a, b, c) .
\end{aligned}
$$

Here, $P(a, b, c)$ is the probability of the system visiting the state with $a$ A molecules, $b \mathrm{~B}$ molecules, and $c \mathrm{C}$ molecules. $P(a, b, c)$ is a function of time as well; however, it is not listed explicitly to simplify the notation. If the reactions that consume species $\mathrm{B}$ are much faster than the reactions that produce $\mathrm{B}$, then species $\mathrm{B}$ is a QSSA species. In solving the master equation, virtually all of the probability for the marginal density of the QSSA species B is in the $b=0$ state, as demonstrated by Fig. 2(a). Also, the probability of the marginal density $P(b)$ decreases exponentially as $b$ increases. In simulation, trajectories of the QSSA species B exhibit shortlived bursts, as demonstrated in Fig. 2(b). These results suggest the existence of a reduced master equation containing only species $\mathrm{A}$ and $\mathrm{C}$ on a slow time scale.
When $\mathrm{B}$ is a QSSA species, $k_{-1}, k_{2} \gg k_{1}$, the small parameter can be written as

$$
\epsilon=\frac{1}{k_{-1}+k_{2}} .
$$

Although we used rate constants as perturbation parameters, the rate constants may not be sufficient to ensure that the QSSA species usually samples zero for all locations of the parameter space. The QSSA species consumption and production rates, which are products of rate constants and species numbers, determine if a species is a QSSA species at any given location in the state space. Depending on the application one may wish to use combinations of rate constants and species initial conditions as the perturbation parameters. For simplicity of exposition, we shall consider only rate constants as the perturbation parameters in this paper.

To apply singular perturbation analysis we write the probability density as a power series in $\epsilon$.

$$
\begin{aligned}
P(a, b, c)= & W_{0}(a, b, c)+\epsilon W_{1}(a, b, c)+\epsilon^{2} W_{2}(a, b, c) \\
& +\cdots .
\end{aligned}
$$

When $\epsilon$ is small (the QSSA limit), $W_{0}(a, b, c)$ is a good approximation of $P(a, b, c) . W_{0}(a, b, c)$ has the properties of a probability density (all terms are greater than zero, and sum to one) because $W_{0}\left(n_{s}, n_{f}\right)=P\left(n_{s}, n_{f}\right)$ when $\epsilon=0$. Our objective in the next section is to write the evolution equation for the first term of this series.

\section{A. Slow time-scale evolution}

The power series [Eq. (10)] is substituted into the master equation [Eq. (9)], and both sides are multiplied by $\epsilon$, to give

$$
\begin{aligned}
\epsilon\left(\frac{d W_{0}}{d t}+\epsilon \frac{d W_{1}}{d t}+\cdots\right)= & \epsilon k_{1}(a+1)\left(W_{0}(a+1, b-2, c)+\epsilon W_{1}(a+1, b-2, c)+\cdots\right)+\frac{1}{2} K_{-1}(b+2)(b+1)\left(W_{0}(a-1, b+2, c)\right. \\
& \left.+\epsilon W_{1}(a-1, b+2, c)+\cdots\right)+K_{2}(b+1)\left(W_{0}(a, b+1, c-1)+\epsilon W_{1}(a, b+1, c-1)+\cdots\right) \\
& -\left(\epsilon k_{1} a+\frac{1}{2} K_{-1} b(b-1)+K_{2} b\right)\left(W_{0}(a, b, c)+\epsilon W_{1}(a, b, c)+\cdots\right)
\end{aligned}
$$

where $K_{i}$ represents $k_{i} \epsilon$ and is of $\mathcal{O}(1)$ or less. Equation (11) can be analyzed by examining the terms grouped by their order in $\epsilon$.

\section{Zero-order terms $\left(\epsilon^{0}\right)$}

Collecting the terms of Eq. (11) that are zero order in $\epsilon$ gives the following equation:

$$
\begin{aligned}
0= & \frac{1}{2} K_{-1}(b+2)(b+1) W_{0}(a-1, b+2, c) \\
& +K_{2}(b+1) W_{0}(a, b+1, c-1) \\
& -\left(\frac{1}{2} K_{-1} b(b-1)+K_{2} b\right) W_{0}(a, b, c) .
\end{aligned}
$$

If the system is closed, then the maximum amount of $B$ in the system, $b_{\max }$, is the sum of the initial amounts of $a$ and $b$. When $b=b_{\max }$ the first two terms of Eq. (12) are not included because $W_{0}\left(a-1, b_{\max }+2, c\right)$ and $W_{0}\left(a, b_{\max }+1\right)$ are zero, and therefore, $W_{0}\left(a-1, b_{\max }, c\right)=0$. This result shows that in the QSSA limit the probability that the QSSA species $B$ reaches its maximum value is zero. This argument is not limited to a closed system. For an open system $b_{\max }$ is bounded by the finite amount of B in the universe.

Given the knowledge that $W_{0}\left(a-1, b_{\max }, c\right)=0$, evaluation of Eq. (12) at $b=b_{\max }-1$ shows that $W_{0}\left(a, b_{\max }-1\right)=0$ also. By successively evaluating Eq. (12) at decreasing 

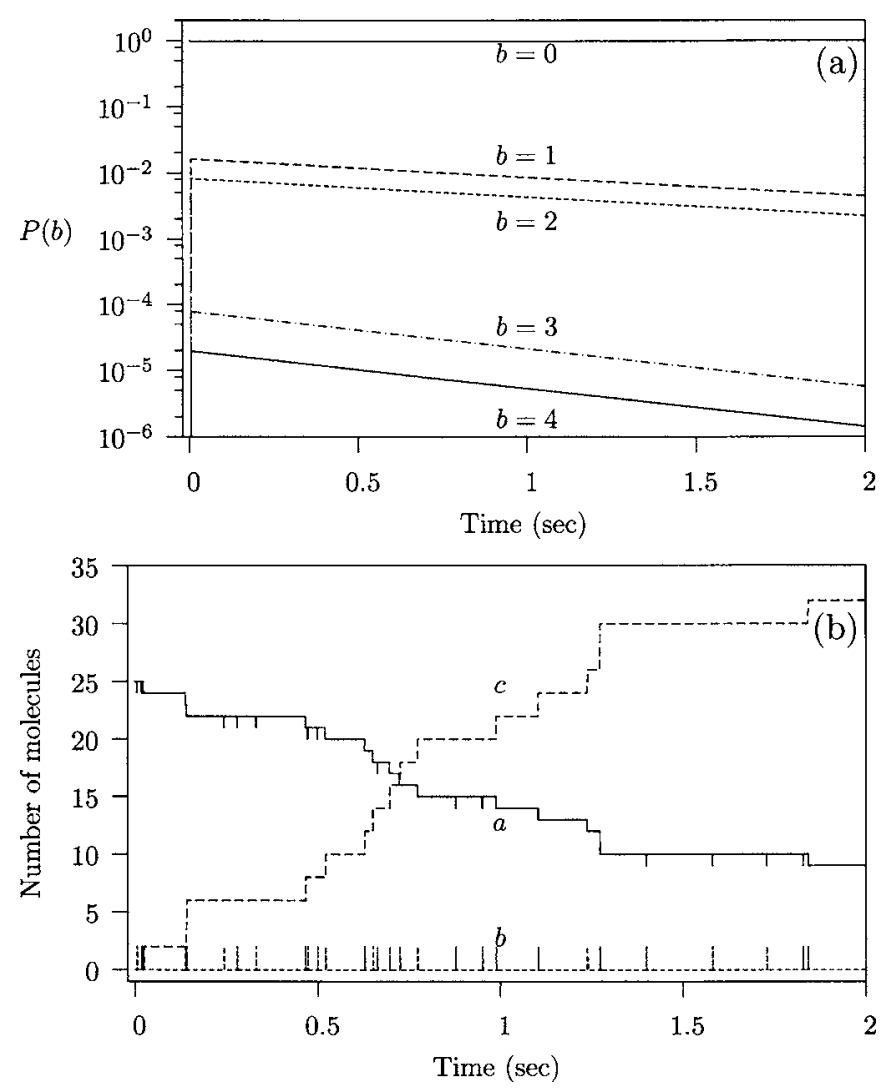

FIG. 2. Solution of reaction (8): (a) evolution of the marginal density $P(b)$ as solved by the master equation, and (b) a single trajectory as solved by exact stochastic simulation. The rate constants $(1 / \mathrm{s})$ are $k_{1}=1, k_{-1}=1000$, and $k_{2}=1000$ and the initial condition is $\left[\begin{array}{lll}a & b & c\end{array}\right]=\left[\begin{array}{lll}25 & 0 & 0\end{array}\right]$.

integer values it follows that $W_{0}(a, b)=0$, for all $b>0$. This result means that to a zero-order approximation, there is zero probability that $B$ ever has a population greater than zero. Therefore, an evolution equation for $W_{0}(a, b, c)$ results in a model where the QSSA species has been removed because the only nonzero terms are $W_{0}(a, 0, c)$.

\section{First-order terms $\left(\epsilon^{1}\right)$}

To derive the evolution equation for $W_{0}(a, 0, c)$, the terms of Eq. (11) that are first order in $\epsilon$ are collected giving the following equation:

$$
\begin{aligned}
\frac{d W_{0}(a, b, c)}{d t}= & k_{1}(a+1) W_{0}(a+1, b-2, c) \\
& +\frac{1}{2} K_{-1}(b+2)(b+1) W_{1}(a-1, b+2, c) \\
& +K_{2}(b+1) W_{1}(a, b+1, c-1) \\
& -k_{1} a W_{0}(a, b, c) \\
& -\left(\frac{1}{2} K_{-1} b(b-1)+K_{2} b\right) W_{1}(a, b, c) .
\end{aligned}
$$

Evaluating Eq. (13) at $b=0$ gives the desired evolution equation

$$
\begin{aligned}
\frac{d W_{0}(a, 0, c)}{d t}= & K_{-1} W_{1}(a-1,2, c)+K_{2} W_{1}(a, 1, c-1) \\
& -k_{1} a W_{0}(a, 0, c) .
\end{aligned}
$$

Equation (14) is not a closed evolution equation because it

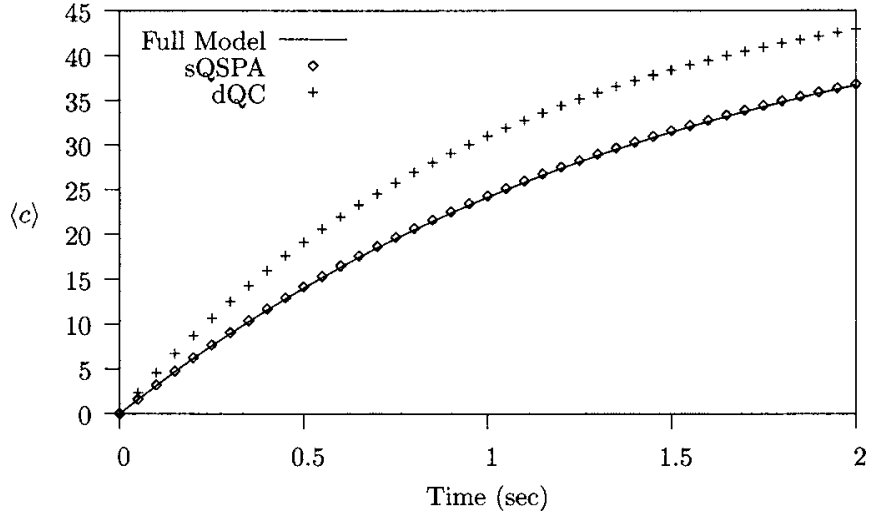

FIG. 3. Comparison of $\langle c\rangle$, calculated by solving the full master equation, the SQSPA master equation, and the reduced master equation with $\mathrm{dQC}$ rate expressions.

depends on the higher-order terms $W_{1}(a, 1, c-1)$ and $W_{1}(a, 2, c)$. The following analysis expresses these higherorder terms in terms of $W_{0}(a, 0, c)$ to close Eq. (14).

By examining Eq. (13) in a similar manner as we examined Eq. (12), starting with $b=b_{\max }$ it can be shown that $W_{1}(a, b, c)=0$, for $b>2$. Evaluating Eq. (13) at $b=2$ and $b$ $=1$ gives Eqs. (15) and (16), respectively,

$$
\begin{aligned}
W_{1}(a, 2, c) & =\frac{k_{1}(a+1)}{K_{-1}+2 K_{2}} W_{0}(a+1,0, c), \\
W_{1}(a, 1, c) & =2 W_{1}(a, 2, c-1) \\
& =\frac{2 k_{1}(a+1)}{K_{-1}+2 K_{2}} W_{0}(a+1,0, c-1) .
\end{aligned}
$$

Substitution of Eqs. (15) and (16) into the evolution equation for $W_{0}(a, 0, c)$ gives the reduced master equation for this system.

$$
\begin{gathered}
\frac{d W_{0}(a, 0, c)}{d t}=\tilde{k}(a+1) W_{0}(a+1,0, c-2)-\tilde{k} a W_{0}(a, 0, c), \\
\tilde{k}=k_{2} k_{1} /\left(k_{-1} / 2+k_{2}\right) .
\end{gathered}
$$

Inspection of this master equation shows that the reaction mechanism for the reduced system is

$$
\mathrm{A} \rightarrow 2 \mathrm{C}, \quad r=\tilde{k} a .
$$

This reduced mechanism can be simulated with SSA, where the sampled probability distribution is the zero-order approximation of the probability distribution from the full master equation.

When dQC is applied to the differential equations that correspond to this reaction mechanism, the stoichiometry of the reduced mechanism is the same. The reaction rate expression is not linear; however,

$$
\mathrm{A} \rightarrow 2 \mathrm{C}, \quad r_{\mathrm{dQC}}=k_{2} \frac{-k_{2}+\sqrt{k_{2}^{2}+8 k_{1} k_{-1} a}}{4 k_{-1}} .
$$

Next we perform a sample calculation on this example system. The full stochastic model, the sQSPA model derived above, and a stochastic model with dQC rate expression are compared. The system initial conditions are $[a b c]$ 


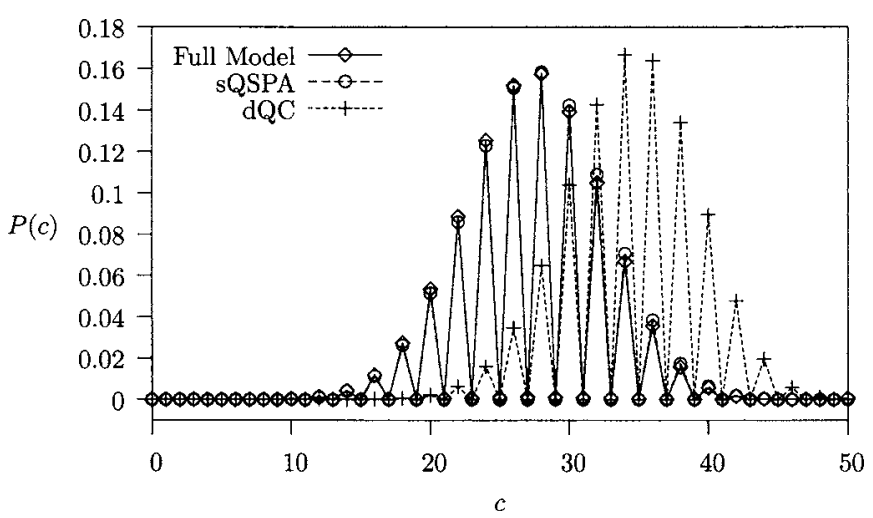

FIG. 4. Comparison of marginal probability densities of $c$ at time $t=1.2 \mathrm{~s}$. The marginal probabilities are calculated by solving the full master equation, the SQSPA master equation, and the reduced master equation with dQC rate expressions.

$=\left[\begin{array}{lll}25 & 0 & 0\end{array}\right]$, and the rate constants $(1 / \mathrm{s})$ are $k_{1}=1$ and $k_{-1}$ $=k_{2}=1000$. These parameters create separation of time scales of two orders of magnitude; therefore, the QSSA approximation should be valid. Figure 3 shows the average evolution of $c(\langle c\rangle)$ calculated by solving the master equation of each model. This figure shows that the deterministic QSSA rate expression does not provide a good approximation of the full system behavior, even though the approximation is valid when ordinary differential equations are used. The stochastic QSSA model does provide an accurate description of the evolution of $\langle c\rangle$.

The derivation above claims not only to provide an accurate approximation of $\langle c\rangle$, but also the probability density $P(a, b, c)$. Figure 4 shows the marginal probability $P(c)$ at $t=1.2 \mathrm{~s}$ for all three models, calculated by solving each master equation. This figure shows that the stochastic QSSA method provides a good approximation for the probability density of the full model.

\section{B. First-order correction}

Given $W_{0}(a, 0, c)$, a first-order correction for the expectation of $b$ can be calculated without solving higher-order evolution equations.

$$
\begin{aligned}
& \langle b\rangle=\sum_{a, b, c} b P(a, b, c), \\
& \langle b\rangle=\sum_{a, b, c} b\left(W_{0}(a, b, c)+\epsilon W_{1}(a, b, c)+\mathcal{O}\left(\epsilon^{2}\right)\right), \\
& \langle b\rangle=\epsilon \sum_{a, c} W_{1}(a, 1, c)+2 W_{1}(a, 2, c)+\mathcal{O}\left(\epsilon^{2}\right) .
\end{aligned}
$$

Since $W_{1}(a, 1, c)$ and $W_{1}(a, 2, c)$ can be expressed in terms of $W_{0}(a, 0, c)$, the first-order approximation of $\langle b\rangle$ can be calculated from $W_{0}(a, 0, c)$.

$$
\langle b\rangle=\frac{2 k_{1}}{k_{-1}+2 k_{2}} \sum_{a} 2 a W_{0}(a, 0, c)+\mathcal{O}\left(\epsilon^{2}\right),
$$

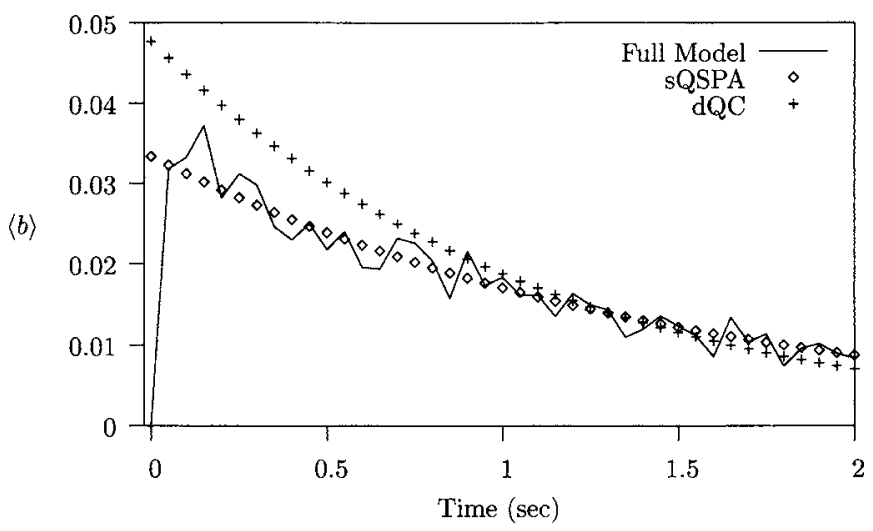

FIG. 5. Comparison of $\langle b\rangle$, calculated from 5000 SSA simulations, with the full model, the sQSPA model, and the dQC model.

$$
\langle b\rangle=\frac{2 k_{1}}{k_{-1} / 2+k_{2}}\langle a\rangle+\mathcal{O}\left(\epsilon^{2}\right) .
$$

In Fig. 5, 5000 SSA simulations are used to calculate $\langle b\rangle$. SSA simulations are used instead of solving the master equation to demonstrate another useful application of reduced models. As seen in Fig. 5, determination of a precise value for $\langle b\rangle$ from simulation of the full model requires many simulations because $b$ is infrequently greater than zero. The sQSPA estimation of $\langle b\rangle$ is smooth after only a few simulations because it is calculated from $\langle a\rangle$, which is calculated precisely with only a few simulations. Notice that the QSSA models calculate $\langle b\rangle$ to be nonzero at $t=0$ because these approximations are valid only on the slow time scale. Additionally, the reduced models executed $75 \%$ less reaction events than the full model, decreasing simulation times by a factor of 4 .

\section{Fast time-scale evolution}

The fast time scale is approximated by substituting the power series expansion of $P(a, b, c)$ into the master equation, but the time scale is changed to $\tau=t / \epsilon$. The power series in $W$ is replaced with $Q$ to emphasize these terms are not equivalent because they apply at different time scales.

The zero-order terms of this series are

$$
\begin{aligned}
\frac{d Q_{0}(a, b, c)}{d \tau}= & \frac{1}{2} K_{-1}(b+2)(b+1) Q_{0}(a-1, b+2, c) \\
& +K_{2}(b+1) Q_{0}(a, b+1, c-1) \\
& -\left(\frac{1}{2} K_{-1} b(b-1)+K_{2} b\right) Q_{0}(a, b, c) .
\end{aligned}
$$

To a zero-order approximation, Eq. (19) shows that on the fast time scale only reactions that consume the QSSA species occur. The steady-state solution to Eq. (19) should be used as the initial condition for the slow time-scale evolution equation. Inspection of Eq. (19) shows that all of the probability flows to the $b=0$ states. Therefore, the steady state of fast time scale is consistent with the slow time-scale requirement that $W_{0}(a, b, c)=0$ for all $b>0$.

In Fig. 6 we compare the solution of the full master equation and the zero-order inner evolution equation [Eq. (19)]. Figure 6 is a reproduction of Fig. 2; however, the time 


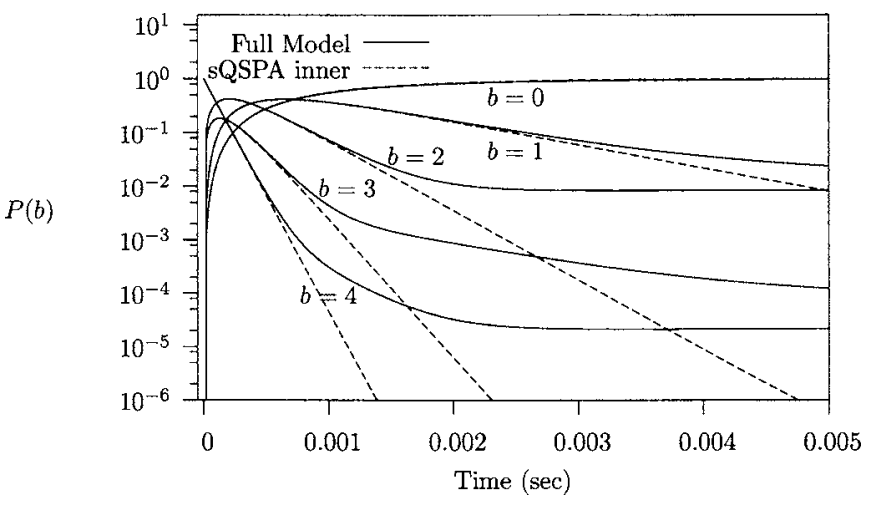

FIG. 6. Solution of reaction (8) on the fast time scale. Evolution of the marginal density $P(b)$ as solved by the master equation, and the zero-order inner sQSPA equation. The rate constants $(1 / \mathrm{s})$ are $k_{1}=1, k_{-1}=1000$, and $k_{2}=1000$ and the initial condition is $\left[\begin{array}{lll}a & b & c\end{array}\right]=\left[\begin{array}{lll}25 & 4 & 0\end{array}\right]$.

scale has been shortened to show the initial transients. Additionally, the initial condition of species B is set to 4 to demonstrate how the probability of $b=4$ decays and $b=0$ rises rapidly as $\mathrm{B}$ is consumed. The probability of the intermediate values of $b$ quickly rise, then decay as the population of $B$ briefly passes through those values on its path to zero. The main distinction between the full master equation solution and $Q_{0}$ equation is that the $Q_{0}$ equation predicts that the marginal probability $b$ approaches zero exponentially for all $b>0$, while the master equation predicts that $P(b)$ approaches small values $[\mathcal{O}(\epsilon)$ or less $]$ for $b>0$.

\section{EXAMPLE TABLE}

Table II lists six examples of full reaction mechanisms and their reduced mechanisms after SQSPA has been applied. This table is intended as a reference for someone reducing a simple mechanism or learning the reduction procedures de- scribed in this paper. In each of these examples only one species is removed; species B for entries 1-4, and species E and ES for entries 5-6, respectively. To remove more than one species from a mechanism the SQSPA procedure can be applied, repeatedly, one species at a time.

The SQSPA and dQC reduced models are equivalent for entries 1-2 and 5-6, but different for entries 3-4. In all six entries, the stoichiometries of the reduced chemical mechanisms for the sQSPA and dQC models are in agreement. This mechanistic agreement is not always the case, as we show in Sec. VI A. Even when the stoichiometries of the reduced chemical mechanisms are in agreement, the reaction rate expressions for the stochastic and deterministic treatments can be quite different as illustrated by entries 3-4.

It is also important to note that the reaction rates that arise from the order reduction do not necessarily obey mass action kinetics of the reduced mechanism. In Table II, entries 1,3 , and 6 follow mass action kinetics of the reduced mechanism; however, entries 2, 4, and 5 do not.

\section{SPA ON A GENERIC REACTION NETWORK}

The purpose of this section is to demonstrate that the procedure for sQSPA, developed for the example in Sec. II, applies generally to a large class of reaction networks. In this section equations that allow a user to directly write down the reduced master equation, for a specific reaction network, without having to work through the perturbation analysis [Eqs. (26)-(28)] are derived.

To accomplish a stochastic QSSA reduction, we first partition the species vector into two sets: $n_{f}$ contains a single QSSA species and $n_{s}$ is vector containing the remaining species

TABLE II. Examples of stochastic QSSA reductions. B is the QSSA species in reaction networks 1-4, and E and ES are the QSSA species in networks 5 and 6 , respectively.

\begin{tabular}{|c|c|c|c|c|c|}
\hline Full mechanism & Rates & $\begin{array}{l}\text { Reduced } \\
\text { mechanism }\end{array}$ & Rates & $\begin{array}{l}\text { Parameters } \\
\text { full/reduced }\end{array}$ & $\begin{array}{c}\text { Stochastic } \\
\text { same as } \\
\text { deterministic? }\end{array}$ \\
\hline $\begin{array}{l}\mathrm{A} \rightleftharpoons \mathrm{B} \\
\mathrm{B} \rightarrow \mathrm{C}\end{array}$ & $\begin{array}{l}r_{1}=k_{1} a, r_{-1}=k_{-1} b \\
r_{2}=k_{2} b\end{array}$ & $\mathrm{~A} \rightarrow \mathrm{C}$ & $r=\left(k_{1} k_{2} / k_{-1}+k_{2}\right) a$ & $3 / 1$ & Yes \\
\hline $\begin{array}{c}2 \mathrm{~A} \rightarrow \mathrm{B} \\
\mathrm{B}+\mathrm{A} \rightarrow \mathrm{C}\end{array}$ & $\begin{array}{l}r_{1}=k_{1} a(a-1) \\
r_{2}=k_{2} a b\end{array}$ & $3 \mathrm{~A} \rightarrow \mathrm{C}$ & $r=k_{1} a(a-1)$ & $2 / 1$ & Yes \\
\hline $\begin{aligned} \mathrm{A} & \rightleftharpoons 2 \mathrm{~B} \\
\mathrm{~B} & \rightarrow \mathrm{C}\end{aligned}$ & $\begin{array}{l}r_{1}=k_{1} a, r_{-1}=k_{-1} / 2 b(b-1) \\
r_{2}=k_{2} b\end{array}$ & $\mathrm{~A} \rightarrow 2 \mathrm{C}$ & $r=\left(k_{1} k_{2} /\left(k_{-1} / 2+k_{2}\right)\right) a$ & $3 / 1$ & No \\
\hline $\begin{array}{c}\mathrm{A} \rightarrow 2 \mathrm{~B} \\
2 \mathrm{~B} \rightarrow \mathrm{D} \\
\mathrm{B}+\mathrm{C} \rightarrow \mathrm{E}\end{array}$ & $\begin{array}{l}r_{1}=k_{1} a \\
r_{2}=k_{2} / 2 b(b-1) \\
r_{3}=k_{3} b c\end{array}$ & $\begin{array}{c}\mathrm{A} \rightarrow \mathrm{D} \\
\mathrm{A}+2 \mathrm{C} \rightarrow 2 \mathrm{E}\end{array}$ & $\begin{array}{l}r_{1}=k_{1} a /\left(1+\left(2 k_{3} / k_{2}\right) c\right) \\
r_{2}=k_{1} a /\left(1+k_{2} /\left(2 k_{3} c\right)\right)\end{array}$ & $3 / 2$ & No \\
\hline $\begin{array}{c}\mathrm{S}+\mathrm{E} \rightleftharpoons \mathrm{ES} \\
\mathrm{ES} \rightarrow \mathrm{P}+\mathrm{E} \\
\text { E small }\left(k_{1} \gg k_{-1}, k_{2}\right.\end{array}$ & $\begin{array}{l}r_{1}=k_{1}(s)(e), r_{-1}=k_{-1}(e s) \\
r_{2}=k_{2}(e s)\end{array}$ & $\mathrm{S} \rightarrow \mathrm{P}$ & $r=k_{2} e_{T}$ & $3 / 1$ & Yes \\
\hline $\begin{array}{c}\mathrm{S}+\mathrm{E} \rightleftharpoons \mathrm{ES} \\
\mathrm{ES} \rightarrow \mathrm{P}+\mathrm{E} \\
\mathrm{ES} \text { small } \\
\left(k_{-1}, k_{2} \gg k_{1}\right)\end{array}$ & $\begin{array}{l}r_{1}=k_{1}(s)(e), r_{-1}=k_{-1}(e s) \\
r_{2}=k_{2}(e s)\end{array}$ & $\mathrm{S} \rightarrow \mathrm{P}$ & $r=\left(k_{2} k_{1} e_{T} / k_{-1}+k_{2}\right) s$ & $3 / 1$ & Yes \\
\hline
\end{tabular}




$$
n=\left[\begin{array}{l}
n_{s} \\
n_{f}
\end{array}\right] .
$$

The stoichiometric matrix is also partitioned into submatrices. The rows are partitioned such that $n_{f}$ is separated from the other species. The columns are partitioned such that reactions that consume $n_{f}$ are marked with a tilde. All the elements of $\widetilde{\nu}_{f} \leqslant-1$, because the QSSA species are consumed in these reactions, and $\nu_{f}=0$.

$$
\nu=\left[\begin{array}{cc}
\nu_{s} & \nu_{f} \\
\widetilde{\nu}_{s} & \widetilde{\nu}_{f}
\end{array}\right] .
$$

There are $N_{f}$ reactions that consume species $n_{f}$ and $N_{s}$ reactions that do not. The partitioned master equation can be written as

$$
\begin{aligned}
\frac{d P\left(n_{s}, n_{f}\right)}{d t}= & \sum_{j=1}^{N_{f}}\left[k_{j} a_{j}\left(n_{s}-\widetilde{\nu}_{s j}, n_{f}-\widetilde{\nu}_{f j}\right) P\left(n_{s}-\widetilde{\nu}_{s j}, n_{f}-\widetilde{\nu}_{f j}\right)\right. \\
& \left.-k_{j} a_{j}\left(n_{s}, n_{f}\right) P\left(n_{s}, n_{f}\right)\right] \\
& +\sum_{i=1}^{N_{s}}\left[k _ { i } a _ { i } ( n _ { s } - \nu _ { s i } , n _ { f } - \nu _ { f i } ) P \left(n_{s}-\nu_{s i}, n_{f}\right.\right.
\end{aligned}
$$

$$
\left.\left.-\nu_{f i}\right)-k_{i} a_{i}\left(n_{s}, n_{f}\right) P\left(n_{s}, n_{f}\right)\right] .
$$

To apply singular perturbation analysis we write the probability density as a power series in $\epsilon$.

$$
P\left(n_{s}, n_{f}\right)=W_{0}\left(n_{s}, n_{f}\right)+\epsilon W_{1}\left(n_{s}, n_{f}\right)+\epsilon^{2} W_{2}\left(n_{s}, n_{f}\right)+\cdots .
$$

$W_{0}\left(n_{s}, n_{f}\right)$ has the properties of a probability density (all terms are greater than zero, and sum to one) because $W_{0}\left(n_{s}, n_{f}\right)=P\left(n_{s}, n_{f}\right)$ when $\epsilon=0$. The perturbation parameter is chosen to be the inverse of the sum of the reaction rate constants that consume species $n_{f}$.

$$
\boldsymbol{\epsilon}=\left(\sum_{j=1}^{N_{f}} k_{j}\right)^{-1} .
$$

\section{A. Slow time-scale evolution}

The long-time approximation is found by substituting this power series into the master equation, then multiplying both sides by $\epsilon$.

$$
\begin{aligned}
\epsilon\left(\frac{d W_{0}}{d t}+\epsilon \frac{d W_{1}}{d t}+\cdots\right)= & \sum_{j=1}^{N_{f}}\left(K_{j} a_{j}\left(n_{s}-\widetilde{\nu}_{s j}, n_{f}-\widetilde{\nu}_{f j}\right)\left(W_{0}\left(n_{s}-\widetilde{\nu}_{s j}, n_{f}-\widetilde{\nu}_{f j}\right)+\cdots\right)-K_{j} a_{j}\left(n_{s}, n_{f}\right)\left(W_{0}\left(n_{s}, n_{f}\right)+\cdots\right)\right) \\
& +\epsilon \sum_{i=1}^{N_{s}}\left(k_{i} a_{i}\left(n_{s}-\nu_{s i}, n_{f}-\nu_{f i}\right)\left(W_{0}\left(n_{s}-\nu_{s i}, n_{f}-\nu_{f i}\right)+\cdots\right)-k_{i} a_{i}\left(n_{s}, n_{f}\right)\left(W_{0}\left(n_{s}, n_{f}\right)+\cdots\right)\right),
\end{aligned}
$$

in which $K_{i}$ represents $k_{i} \epsilon$ and is of $\mathcal{O}(1)$ or less. Reduced models are be identified from Eq. (23) by examining the terms grouped by their order in $\epsilon$.

\section{Zero-order terms $\left(\epsilon^{0}\right)$}

Collecting the terms that are zero order in $\epsilon$ from Eq. (23) gives the following equation:

$$
\begin{aligned}
0= & \sum_{j=1}^{N_{f}} K_{j} a_{j}\left(n_{s}-\widetilde{\nu}_{s j}, n_{f}-\widetilde{\nu}_{f j}\right) W_{0}\left(n_{s}-\widetilde{\nu}_{s j}, n_{f}-\widetilde{v}_{f j}\right) \\
& -K_{j} a_{j}\left(n_{s}, n_{f}\right) W_{0}\left(n_{s}, n_{f}\right) .
\end{aligned}
$$

In the same manner as in Sec. II A 1, application of a finitemass constraint can be used to show that $W_{0}\left(n_{s}, n_{f}\right)=0$ if any of the consumption rates $a_{j}\left(n_{s}, n_{f}\right)$ are nonzero. Under conditions of elementary, bimolecular and unimolecular reactions, this ensures that $W_{0}\left(n_{s}, n_{f}\right)=0$ for $n_{f} \geqslant 2$. In most cases $W_{0}\left(n_{s}, 0\right)$ is the only nonzero portion of $W\left(n_{s}, n_{f}\right)$. Therefore, to a zero-order approximation, QSSA species have a population of zero.

In the case that the only reactions that consume $n_{f}$ consume two $n_{f}$ molecules, then $W_{0}\left(n_{s}, 1\right)$ is also nonzero. In this case, the QSSA species can have a population of 0 or 1 , but as soon as the population reaches 2, the QSSA species react to bring the population back down to 0 . This situation is discussed in more detail in Sec. VI B.

\section{First-order terms $\left(\epsilon^{1}\right)$}

Collecting the terms that are first order in $\epsilon$ gives the following equation:

$$
\begin{aligned}
\frac{d W_{0}\left(n_{s}, n_{f}\right)}{d t}= & \sum_{j=1}^{N_{f}} K_{j} a_{j}\left(n_{s}-\widetilde{\nu}_{s j}, n_{f}-\widetilde{\nu}_{f j}\right) W_{1}\left(n_{s}-\widetilde{\nu}_{s j}, n_{f}-\widetilde{\nu}_{f j}\right) \\
& -K_{j} a_{j}\left(n_{s}, n_{f}\right) W_{1}\left(n_{s}, n_{f}\right) \\
& +\sum_{i=1}^{N_{s}} k_{i} a_{i}\left(n_{s}-\nu_{s i}, n_{f}-\nu_{f i}\right) W_{0}\left(n_{s}-\nu_{s i}, n_{f}-\nu_{f i}\right) \\
& -k_{i} a_{i}\left(n_{s}, n_{f}\right) W_{0}\left(n_{s}, n_{f}\right) .
\end{aligned}
$$

For the purpose of this analysis we assume that $W_{0}\left(n_{s}, n_{f}\right)$ $=0$ for $n_{f} \geqslant 1$. The evolution equation of $W_{0}\left(n_{s}, 0\right)$ is 


$$
\begin{aligned}
\frac{d W_{0}\left(n_{s}, 0\right)}{d t}= & \sum_{j=1}^{N_{f}} K_{j} a_{j}\left(n_{s}-\widetilde{\nu}_{s j},-\widetilde{\nu}_{f j}\right) W_{1}\left(n_{s}-\widetilde{\nu}_{s j},-\widetilde{\nu}_{f j}\right) \\
& +\sum_{i=1}^{N_{s}} k_{i} a_{i}\left(n_{s}-\nu_{s i},-\nu_{f i}\right) W_{0}\left(n_{s}-\nu_{s i},-\nu_{f i}\right) \\
& -k_{i} a_{i}\left(n_{s}, 0\right) W_{0}\left(n_{s}, 0\right) .
\end{aligned}
$$

As written, Eq. (26) does not close in $W_{0}\left(n_{s}, 0\right)$ because $W_{1}\left(n_{s}, n_{f}\right)$ terms are needed. The objective of the following analysis is to express the nonzero terms of $W_{1}\left(n_{s}, n_{f}\right)$ in terms of $W_{0}\left(n_{s}, 0\right)$. The expression for $W_{1}\left(n_{s}, p\right)$ and $W_{0}\left(n_{s}, q\right)$ can be substituted into Eq. (26) to give the evolution equation for the zero-order approximation of the probability density. This zero-order approximation is the reduced model of interest.

Just as many of the $W_{0}\left(n_{s}, n_{f}\right)$ terms were shown to be zero from the zero-order equation, we can show that $W_{1}\left(n_{s}, n_{f}\right)=0$ for $n_{f} \geqslant p+1$, in which $p$ is equal to the maximum number of QSSA species produced in a single reaction. In the example given in Sec. II A 2, $p=2$. In most reaction networks $p$ is not greater than 2 .

Analyzing Eq. (25) at $n_{f}=p$ we obtain

$$
W_{1}\left(n_{s}, p\right)=\frac{\sum_{i=1}^{N_{s}} k_{i} a_{i}\left(n_{s}-\nu_{s i}, p-\nu_{f i}\right) W_{0}\left(n_{s}-\nu_{s i}, p-\nu_{f i}\right)}{\sum_{j=1}^{N_{f}} K_{j} a_{j}\left(n_{s}, p\right)} .
$$

Equation (27) is analogous to Eq. (15) in the example derivation. When $n_{f}=q$, where $q=1, \ldots, p-1$, the following expression for $W_{0}\left(n_{s}, q\right)$ can be generated from Eq. (25):

$$
\begin{aligned}
& W_{1}\left(n_{s}, q\right)=\frac{\sum_{i=1}^{N_{s}} k_{i} a_{i}\left(n_{s}-\nu_{s i}, q-\nu_{f i}\right) W_{0}\left(n_{s}-\nu_{s i}, q-\nu_{f i}\right)}{\sum_{j=1}^{N_{f}} K_{j} a_{j}\left(n_{s}, q\right)} \\
& +\frac{\sum_{j=1}^{N_{f}} K_{j} a_{j}\left(n_{s}-\widetilde{\nu}_{s j}, q-\widetilde{\nu}_{f j}\right) W_{1}\left(n_{s}-\widetilde{\nu}_{s j}, q-\widetilde{\nu}_{f j}\right)}{\sum_{j=1}^{N_{f}} K_{j} a_{j}\left(n_{s}, q\right)} .
\end{aligned}
$$

Equation (28) is analogous to Eq. (16) in the example derivation.

Equations (27) and (28) provide the nonzero portions of $W_{1}\left(n_{s}, n_{f}\right)$ in terms of $W_{0}\left(n_{s}, 0\right)$. The intuitive explanation of these equations is that once a QSSA species is generated (i.e., $n_{f}>0$ ), it is immediately eliminated by reactions consuming the QSSA species to make $n_{f}=0$. Consequently, substituting Eqs. (27) and (28) into the evolution equation of $W_{0}\left(n_{s}, 0\right)$ yields a closed set of differential equations for $W_{0}\left(n_{s}, 0\right)$. That is, the reduced master equation consists solely of slow species. By inspection, one can deduce the reduced rate expressions and stoichiometries from this reduced master equation; the reduced mechanisms presented in Table II were obtained in this fashion. The resulting evolution equation for $W_{0}\left(n_{s}, 0\right)$ can be solved as a set of differential equations or simulated with SSA, and the solution represents the zero-order approximation of $P\left(n_{s}, n_{f}\right)$.

\section{B. First-order correction}

A first-order correction to the expectation of $n_{f}$ can be calculated given $W_{0}\left(n_{s}, 0\right)$.

$$
\begin{aligned}
\left\langle n_{f}\right\rangle & =\sum_{n_{s}, n_{f}} n_{f} P\left(n_{s}, n_{f}\right) \\
& =\sum_{n_{s}, n_{f}} n_{f}\left(W_{0}\left(n_{s}, n_{f}\right)+\epsilon W_{1}\left(n_{s}, n_{f}\right)+\mathcal{O}\left(\epsilon^{2}\right)\right) \\
& =\epsilon \sum_{n_{s}} \sum_{n_{f}=1}^{p} n_{f}\left(W_{1}\left(n_{s}, n_{f}\right)+\mathcal{O}\left(\epsilon^{2}\right)\right) .
\end{aligned}
$$

Since $W_{1}\left(n_{s}, n_{f}\right)$ can be expressed in terms of $W_{0}\left(n_{s}, 0\right)$, the first-order approximation of $\left\langle n_{f}\right\rangle$ can be calculated from $W_{0}\left(n_{s}, 0\right)$. If $p=1$, then

$$
\begin{aligned}
& \left\langle n_{f}\right\rangle=\epsilon \sum_{n_{s}} \frac{\sum_{i=1}^{N_{s}} k_{i} a_{i}\left(n_{s}-\nu_{s i}, 1-\nu_{f i}\right) W_{0}\left(n_{s}-\nu_{s i}, 1-\nu_{f i}\right)}{\sum_{j=1}^{N_{f}} K_{j} a_{j}\left(n_{s}, 1\right)} \\
& +\mathcal{O}\left(\epsilon^{2}\right) \text {. }
\end{aligned}
$$

\section{Fast time-scale evolution}

The fast time scale is approximated by substituting the power series expansion of $P\left(n_{s}, n_{f}\right)$ into the generic master equation and changing the time scale to $\tau=t / \epsilon$. The analysis of the fast time scale of the generic reaction network is similar to the example given is Sec. II C; therefore, the mathematics are not repeated here.

This analysis shows that on the fast time scale only reactions that consume QSSA species occur; therefore, the QSSA species is completely consumed during the initial transient. The steady-state solution of the fast-time scale evolution equation is consistent with the slow time-scale requirement that $W_{0}\left(n_{s}, n_{f}\right)=0$ for all $n_{f}>0$. In the case that the only reactions that consume $n_{f}$ consume two $n_{f}$ molecules, $Q_{0}\left(n_{s}, 1\right)$ also has nonzero probability at steady state.

\section{MICHAELIS-MENTEN KINETICS}

A commonly applied example of deterministic QSSA is enzyme kinetics. In this reaction network unbound enzyme $\mathrm{E}$ reversibly binds with a substrate $S$ to form the enzymesubstrate complex ES. The product $\mathrm{P}$ is then formed upon the decomposition of ES.

$$
\begin{aligned}
& \mathrm{E}+\underset{k_{-} 1}{\stackrel{k_{1}}{\rightleftharpoons}} \mathrm{ES}, \\
& \mathrm{ES} \stackrel{k_{2}}{\longrightarrow} \mathrm{P}+\mathrm{E} .
\end{aligned}
$$

Henri first showed how this mechanism could be reduced using the reaction equilibrium assumption in 1902. ${ }^{23}$ Michaelis and Menten later used Henri's work to demonstrate how kinetic parameters $\left(V_{\max }, K_{m}\right)$ can be obtained from experimental data in their 1913 paper. ${ }^{24,25}$ In 1925 Briggs and Haldane used a dQC method to reduce the mechanism. ${ }^{26}$ In both the reaction equilibrium and dQC approximations the production rate equation of $\mathrm{P}$ has the same functional form [Eq. (31)], although the evolution equations for the other species differ. The dQC mechanism is 


$$
\mathrm{S} \rightarrow \mathrm{P}, \quad r=\frac{V_{\max } c_{s}}{K_{m}+c_{s}},
$$

in which $V_{\max }=k_{2} e_{T}, K_{m}=\left(k_{-1}+k_{2}\right) / k_{1}$, and $e_{T}$ is the total amount of enzyme.

In 1967 Heineken et al. showed that the above reduced mechanism is also derived from the zero-order equations from dQSPA. ${ }^{27}$ They also derived a model with higher-order corrections for this mechanism.

Enzyme reactions arise frequently in biological reaction networks where stochastic representations are important. Therefore, it is important to know if the Michaelis-Menten rate expression arises when a QSSA reduction is applied to the master equation.

As shown in Table II the sQSPA method can be applied to the enzyme kinetics mechanism in two distinct ways, either E or ES can be assumed to be the QSSA species. dQC results in the same reduced stoichiometry and rate expression when applied to either E or ES. When sQSPA is applied the following rate expressions for the $\mathrm{S} \rightarrow \mathrm{P}$ reaction result when ES and E are the QSSA species.

$$
\begin{aligned}
& \text { sQSPA E small, } \quad r=V_{\max }, \\
& \text { sQSPA ES small, } \quad r=\frac{V_{\max } S}{K_{m}} .
\end{aligned}
$$

Notice that the Michaelis-Menten rate expression did not explicitly arise from the sQSPA model reduction. However, the sQSPA rate expressions are limiting cases of the MichaelisMenten rate expression. In the case of E small and $K_{m} \ll 1$, the Michaelis-Menten rate expression becomes a constant $V_{\max }$, equivalent to the sQSPA rate expression. In the limit of $K_{m} \gg 1$, when ES is small, the Michaelis-Menten rate expression is linear in $a$, again equivalent to the SQSPA rate expression in Table II. The Michaelis-Menten model is therefore a valid approximation of the full stochastic model when either $\mathrm{E}$ or ES is nearly zero.

It is well documented in the deterministic setting that the Michaelis-Menten model is valid only in certain portions of the parameter and state space. Schnell and Maini stated that the Michaelis-Menten model is valid when $s_{0} \gg e_{0}$ or $K_{m}$ $\gg e_{0}{ }^{28}$ Segel and Slemrod developed another criteria for the validity of the Michaelis-Menten model, that $e_{0} /\left(K_{m}+s_{0}\right)$ is approximately the fractional error in the estimate of $s .{ }^{29}$ By moving from a deterministic to a stochastic system, the Michaelis-Menten mechanism does not somehow become valid in regions where the deterministic analog is invalid.

These criteria for the validity of the Michaelis-Menten models in deterministic settings are not generally applicable for testing the validity of Michaelis-Menten models in stochastic settings. In the Introduction we imposed two requirements for the validity of reduced models: (i) the reduced models need to be simpler than the full model, and (ii) the solutions of the reduced models need to converge to the full model as a specified parameter goes to zero. SQSPA model reductions show that Michaelis-Menten rate expressions satisfy requirement (ii) when either E or ES has a population of zero the majority of the time.

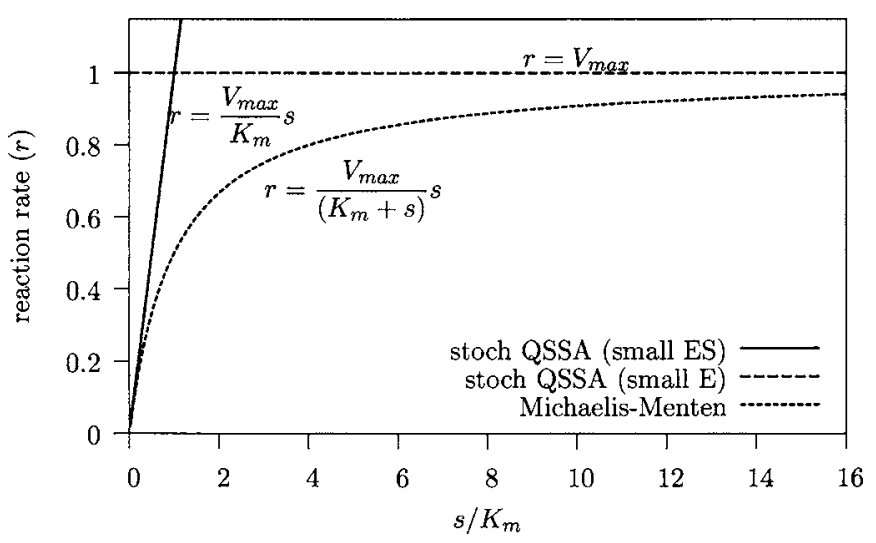

FIG. 7. Comparison of the sQSPA rate expressions and the MichaelisMenten rate expression $\left(V_{\max }=1 \mathrm{~s}^{-1}, s=2\right)$. Both when $s / K_{m}$ is large and when $s / K_{m}$ is small, the Michaelis-Menten rate expression matches one of the sQSPA expressions.

In Fig. 7 we plot the Michaelis-Menten rate expression and the two limiting expressions from sQSPA. When $s \approx e$ $\approx 1$ and $s /\left(K_{m}\right)=1$, the fractional error prediction established by Segel and Slemrod is approximately 1; therefore, in the deterministic setting the Michaelis-Menten rate expression is invalid. There is no reason to believe that the MichaelisMenten rate expression will be valid in stochastic kinetics in this region. In limiting regions of $\left(s / K_{m}\right)$ the MichaelisMenten expression is as appropriate for use in stochastic simulations as the sQSPA expressions derived in this paper.

\section{SPECIAL CASES}

\section{A. Catalytic reactions}

Consider the following reaction mechanism with elementary reaction rates:

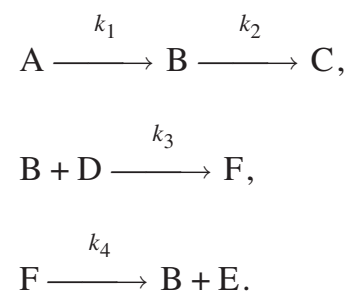

If $k_{4} \gg k_{3}$, then sQSPA as described previously can be applied to remove species $F$. The following mechanism results, with elementary reaction rates.

$$
\begin{aligned}
& \mathrm{A} \stackrel{k_{1}}{\longrightarrow} \mathrm{B} \stackrel{k_{2}}{\longrightarrow} \mathrm{C}, \\
& \mathrm{B}+\mathrm{D} \stackrel{k_{3}}{\longrightarrow} \mathrm{B}+\mathrm{E} .
\end{aligned}
$$

The final reaction rate of this reduced mechanism is now a function of $b$. The stoichiometric number for $\mathrm{B}$ in this reaction is zero, however. Species B acts as a catalyst in this final reaction. If $k_{2}, k_{3} \gg k_{1}$, then the singular perturbation analysis described earlier can be applied to remove B from the reaction mechanism. In this section, we describe how to apply the singular perturbation analysis to remove a QSSA species that acts as catalyst for other reactions. This situation often 


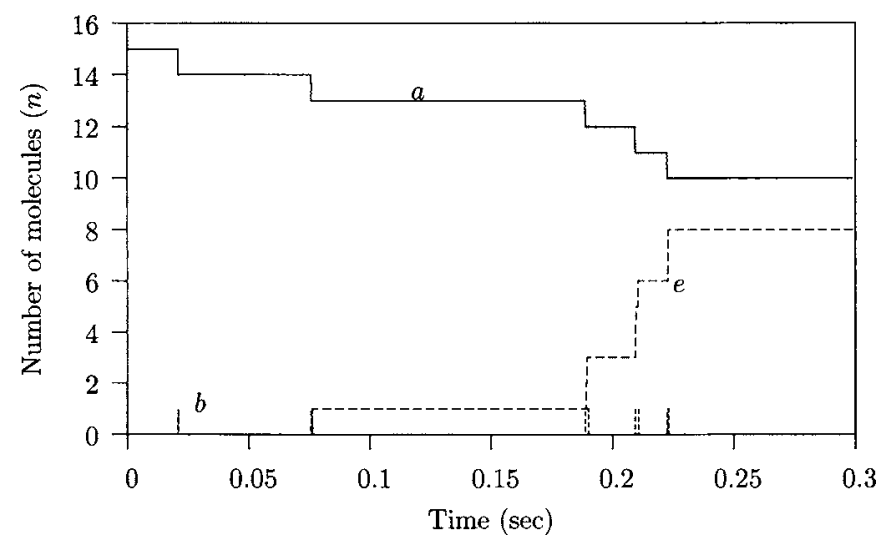

FIG. 8. Single realization to mechanism (32). The rate constants $(1 / \mathrm{s})$ are $k_{1}=1, k_{2}=1000, k_{3}=100$. The initial conditions are $a=15, d=20$, and $b=c$ $=e=0$.

arises when removing more than one species from a reaction mechanism.

Figure 8 is an illustration of what may happen in a stochastic simulation of the above mechanism (32) when $\mathrm{B}$ is a QSSA species. We see that soon after B is formed it quickly decomposes to $\mathrm{C}$. Since the catalyst reaction can occur only when $\mathrm{B}$ is greater than zero, $\mathrm{E}$ is produced only during these short time intervals. Figure 8 shows that after a B molecule is produced, there is a possibility that the catalytic reaction does not happen $(t=0.02 \mathrm{~s})$, happens once $(t=0.07 \mathrm{~s})$, or happens several times $(t=0.18,0.21,0.22 \mathrm{~s})$ before $\mathrm{B}$ is consumed.

A method that removes the QSSA species from the model should correctly capture the dynamics of the remaining species. Therefore, a reduced model for this system should allow the catalytic reaction to not occur, occur once, or occur many times after the initiating reaction $(\mathrm{A} \rightarrow \mathrm{C})$ takes place. We show below that the perturbation analysis does create a reduced model with this feature. To obtain the evolution equation for the zero-order approximation of the probability density of reaction network (32), we apply Eq. (26), where $\epsilon=1 / k_{2}$, and $K_{3}=k_{3} \epsilon$.

$$
\begin{aligned}
\frac{d W_{0}(a, b, c, d, e)}{d t}= & k_{1}(a+1) W_{0}(a+1, b-1, c, d, e) \\
& +(b+1) W_{1}(a, b+1, c-1, d, e) \\
& +K_{3} b(d+1) W_{1}(a, b, c, d+1, e-1) \\
& -k_{1} a W_{0}(a, b, c, d, e) \\
& -\left(b+K_{3} b d\right) W_{1}(a, b, c, d, e) .
\end{aligned}
$$

The terms in Eq. (33) that correspond to the catalytic reaction are multiplied by $W_{1}$ instead of $W_{0}$ because the catalytic reaction occurs on the fast time scale.

Evaluating Eq. (33) at $b=0$ gives the reduced system's master equation.

$$
\begin{aligned}
\frac{d W_{0}(a, 0, c, d, e)}{d t}= & W_{1}(a, 1, c-1, d, e) \\
& -k_{1} a W_{0}(a, 0, c, d, e) .
\end{aligned}
$$

To remove the $W_{1}$ term from the reduced master equation, Eq. (33) needs to be evaluated at $b=1$.

$$
\begin{aligned}
W_{1}(a+1,0, c, d, e)= & \frac{k_{1}(a+1)}{1+K_{3} d} W_{0}(a+1,0, c, d, e) \\
& +\frac{K_{3}(d+1)}{1+K_{3} d} W_{1}(a, 1, c, d+1, e-1) .
\end{aligned}
$$

A single substitution of Eq. (35) into the reduced master equation creates a new $W_{0}$ and a new $W_{1}$ term; therefore, the reduced master equation is not closed. To remove the new $W_{1}$ term the substitution can be performed again. With every substitution a new $W_{0}$ term is created. The first $W_{0}$ term corresponds to the initiating reaction $(\mathrm{A} \rightarrow \mathrm{C})$ without a catalyst reaction $(\mathrm{D} \rightarrow \mathrm{E})$. The second $W_{0}$ term corresponds to an initiating reaction with a single simultaneous catalyst reaction. The $n$th $W_{0}$ term corresponds to an initiating reaction, with $n-1$ simultaneous catalyst reactions. To remove all of the $W_{1}$ terms from the master equation, Eq. (35) has to be recursively substituted until a mass constraint that eliminates the final $W_{1}$ term is reached.

The final reaction mechanism generated from this reduced master equation is

$$
\begin{aligned}
& \mathrm{A} \rightarrow \mathrm{C}, \quad r_{0}=\frac{k_{1} a}{1+K_{3} d}, \\
& \mathrm{D}+\mathrm{A} \rightarrow \mathrm{E}+\mathrm{C}, \quad r_{1}=r_{0} \frac{K_{3} d}{1+K_{3}(d-1)}, \\
& 2 \mathrm{D}+\mathrm{A} \rightarrow 2 \mathrm{E}+\mathrm{C}, \quad r_{2}=r_{1} \frac{K_{3}(d-1)}{1+K_{3}(d-2)}, \\
& \cdots \\
& n \mathrm{D}+\mathrm{A} \rightarrow n \mathrm{E}+\mathrm{C}, \quad r_{n}=r_{n-1} \frac{K_{3}(d+1-n)}{1+K_{3}(d-n)} .
\end{aligned}
$$

There are $m$ possible reactions in this list because the $m+1$ reaction would violate a finite mass constraint. The rate of the $m$ th reaction is calculated in the following manner to ensure probability conservation in the reduced master equation:

$$
r_{m}=k_{1} a-\sum_{n=0}^{m-1} r_{n} .
$$

When dQC is applied to mechanism (32) the following mechanism and reaction rates result.

$$
\begin{aligned}
& \mathrm{A} \rightarrow \mathrm{C}, \quad r_{0}=k_{1} a, \\
& \mathrm{D}+\mathrm{A} \rightarrow \mathrm{E}+\mathrm{A}, \quad r_{1}=\frac{k_{3} k_{1}}{k_{2}} a d .
\end{aligned}
$$

In this case both the stoichiometry and reaction rates of the reduced mechanism from SQSPA and dQC are different.

The number of reactions contained in the reduced master equation increases significantly when a catalytic reaction is 


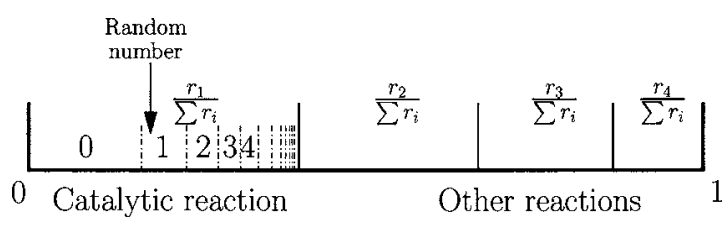

FIG. 9. Schematic that illustrates which reaction is selected in a SSA simulation. The bin that a uniform random number lands in corresponds to the reaction to be executed. The numbers in the first bin correspond to the number of catalytic reactions that are executed along with the initiating reaction.

involved. When sampling this reduced master equation with SSA, all of the additional catalytic reaction rates do not have to be calculated, however. The following discussion shows how SSA can be implemented efficiently when catalytic reactions of the type shown in the above reduced mechanism occur.

During each iteration of the SSA sampling procedure, the time step must be calculated and the next reaction must be chosen. The time step is calculated by sampling a number from an exponential distribution, where the mean of the distribution is the sum of the reaction rates. Explicit calculation of the rates of all of the catalytic reactions is not necessary because their sum is given explicitly in the master equation. For example, in the mechanism given above $\Sigma_{i} r_{i}=k_{1} a$, or the rate of the initiating reaction uncoupled to catalytic reactions.

The procedure for choosing which reaction to implement is illustrated in Fig. 9. A uniformly distributed random number between zero and one is used to select which reaction fires. In Fig. 9 the width of each bin is proportional to each normalized reaction rate. The bin in which the random number falls determines which reaction is executed. If the random number falls in a catalytic reaction bin $\left(r_{1}\right)$, then the number of catalytic reactions that fire along with the initiating reaction needs to be determined. The catalytic reaction rates are then evaluated recursively until their sum is greater than the random number.

To provide a check of the validity of the reduction of the master equation with catalytic reactions, we ran 50000 simulations of sQSPA mechanism [Eq. (37)], the full mechanism [Eq. (32)], and the dQC mechanism [Eq. (37)]. Figure 10 shows a comparison of the marginal probability distributions of $e$ at several times during the simulations. The distributions for the full model and the SQSPA are virtually indistinguishable. This example clearly shows the failure of a model based on a dQC reduction to reproduce the full model behavior. In this example sQSPA required $80 \%$ fewer reaction events than simulation of the full model. While computational improvements are important, this model reduction also reduced the number of parameters from three to two. Additionally, the two kinetic parameters in the reduced model $\left(k_{1}\right.$ and $\left.k_{3} / k_{2}\right)$ are $\mathcal{O}(1)$, and can be estimated from measurements taken on the slow time scale. From these simulations, it is also evident that the dQC mechanism and rate expressions do not provide a good approximation of the full system behavior.

\section{B. Bimolecular consumption of QSSA species}

In the previous examples, the QSSA species virtually always has a population of zero because as soon as the

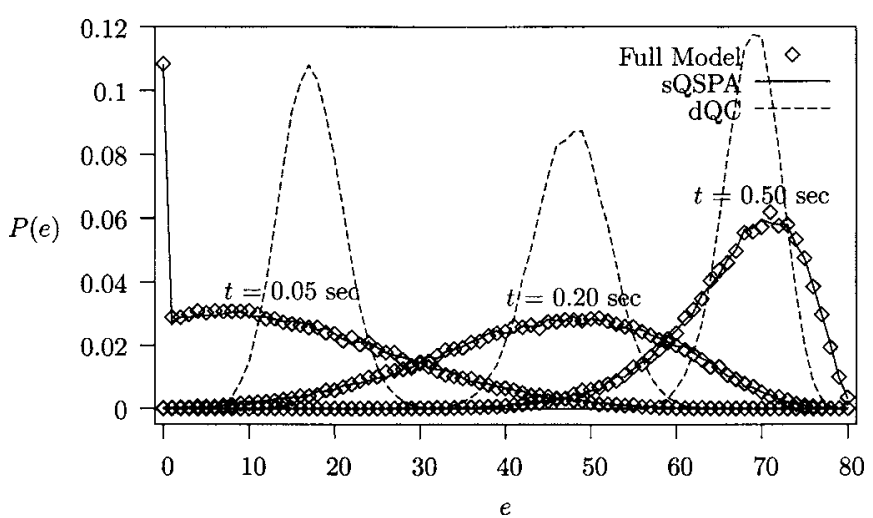

FIG. 10. Comparison of the probability distribution of $e$ calculated from 50000 SSA simulations, with the full master equation, sQSPA, and dQC. Probabilities are computed at times $0.05,0.2$, and $0.5 \mathrm{~s}$. The rate constants $(1 / \mathrm{s})$ are $k_{1}=1, k_{2}=1000$, and $k_{3}=100$. The initial conditions are $a=50, d$ $=80$, and $b=c=e=0$

QSSA species is produced it is eliminated by a reaction consuming it. Therefore the zero-order approximation of the probability density $\left(W_{0}\left(n_{s}, n_{f}\right)\right)$ is zero for all $n_{f}>0$. If the reaction consuming the QSSA species requires two QSSA reactants, then the QSSA species can maintain a population of both 0 and 1 for extended periods of time. A simple reaction mechanism that fits this description is

$$
\begin{aligned}
& \mathrm{A} \stackrel{k_{1}}{\longrightarrow} \mathrm{B}, \\
& \mathrm{2} \mathrm{B} \stackrel{k_{2}}{\longrightarrow} \mathrm{C} .
\end{aligned}
$$

If $k_{2} \gg k_{1}$ then B is a QSSA species; however, two successive slow reactions are required before the reaction eliminating $\mathrm{B}$ can occur. Investigation of the zero-order equations in Sec. IV A 1 for this reaction mechanism show that both $W_{0}(a, 0, c)$ and $W_{0}(a, 1, c)$ are nonzero.

Application of Eq. (25) to this reaction network gives the following evolution equations for $W_{0}(a, 0, c)$ and $W_{0}(a, 1, c)$ :

$$
\begin{aligned}
\frac{d W_{0}(a, 0, c)}{d t}= & k_{1}(a+1) W_{0}(a+1,1, c-1) \\
& -k_{1} a W_{0}(a, 0, c), \\
\frac{d W_{0}(a, 1, c)}{d t}= & k_{1}(a+1) W_{0}(a+1,0, c)-k_{1} a W_{0}(a, 1, c) .
\end{aligned}
$$

These evolution equations show that species $B$ has not been completely removed from the model; however, it has been restricted to two states. These evolution equations also correspond to the following reduced model and reaction rates:

$$
\begin{aligned}
& \mathrm{A} \rightarrow \mathrm{B}, \quad r_{1}=k_{1} a(1-b), \\
& \mathrm{A}+\mathrm{B} \rightarrow \mathrm{C}, \quad r_{2}=k_{1} a b .
\end{aligned}
$$

The species number $b$ acts as a switching function to determine which reaction can fire next. The first reaction can fire only when $b=0$ because additional creation of species $\mathrm{B}$ would put its population above 1 . The second reaction can occur only when $b=1$ because species $\mathrm{C}$ can be created by a reaction only when a B molecule is already present. Simula- 


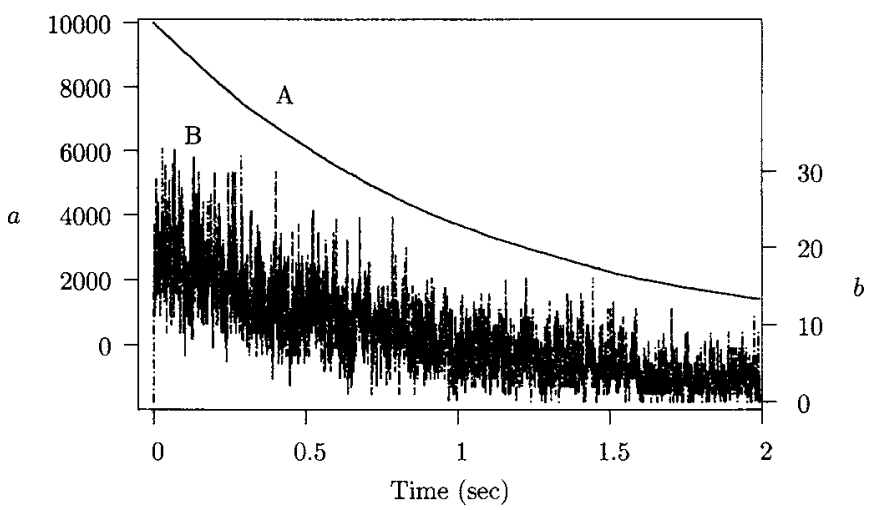

FIG. 11. The species number evolution of $A$ and $B$ from a single SSA simulation, reacting according to mechanism (38) and the parameters listed in this section.

tions show that when $\epsilon$ is sufficiently small, the probability density generated from the full model and the sQSPA model of this system are virtually identical, similar to the results in Figs. 4 and 10.

\section{SPA AND $\Omega$-EXPANSION REDUCTION OF THE MASTER EQUATION}

The example reaction mechanism used in Sec. II is

$$
\begin{aligned}
& \underset{k_{-1}}{\mathrm{~A}} \underset{\mathrm{k}_{1}}{\rightleftharpoons} \mathrm{B}, \quad r_{1}=k_{1} a, \quad r_{-1}=\frac{1}{2 \Omega} k_{-1} b(b-1) \\
& \mathrm{B} \stackrel{k_{2}}{\longrightarrow} \mathrm{C}, \quad r_{2}=k_{2} b .
\end{aligned}
$$

The parameter that measures the size of a chemically reacting system is $\Omega$. The factor of $1 / \Omega$ was added to the reverse reaction rate to account for the volume dependence of bimolecular reactions. This factor was left out in Sec. II due to the assumption that in the stochastic limit $\Omega$ is $\mathcal{O}(1)$. For this example we maintain the rate constants at $k_{1}=11 / \mathrm{s}$ and $k_{-1}=k_{2}=10001 / \mathrm{s}$; however, we change the initial conditions to $\left[a_{0} b_{0} c_{0}\right]=\left[\begin{array}{lll}10^{4} & 0 & 0\end{array}\right]$ and set $\Omega=1000$.

Performing a SSA simulation of this system we observe the trajectories for $a$ and $b$ given in Fig. 11. Notice that $A$ evolves almost deterministically since it has high population values, while the population the QSSA species $B$ remains small and stochastic. The value of $b$ is not usually zero; therefore, the sQSPA technique developed in this paper is not appropriate to reduce this reaction mechanism at these conditions.
When the size of the system becomes large, an approximation for the master equation can be obtained with the $\Omega$ expansion. ${ }^{19,30}$ This expansion is based on the insight that variations in particle numbers are proportional to $\Omega^{1 / 2}$ in the limit of large $\Omega$. In this expansion the following variable transformation from the discrete particle numbers $(n)$ to continuous variables $\phi$ and $\xi$ is performed

$$
n=\Omega \phi+\Omega^{1 / 2} \xi .
$$

The terms of the master equation are then expanded with a Taylor series in powers of $\Omega^{1 / 2}$. The highest-order $\Omega$ terms of this expansion give deterministic evolutions equations for $\phi$. The next highest-order terms give a linear Fokker-Planck equation for $\xi$.

In this section, we apply the $\Omega$ expansion as outlined by van Kampen ${ }^{30}$ with a few modifications for application to QSSA. Instead of applying the variable transformation in Eq. (39) to all of the species, it is only applied to the non-QSSA species; therefore, the QSSA species numbers remain discrete. Additionally the product of the SPA parameter $\epsilon$ and system size $\Omega$ is considered to be $\mathcal{O}(1)$ and therefore changes which terms are retained or discarded at each order in the $\Omega$ expansion.

Reaction mechanism (38) corresponds to the following master equation:

$$
\begin{aligned}
\frac{d P(a, b)}{d t}= & k_{1}(a+1) P(a+1, b-2) \\
& +\frac{1}{2} k_{-1}(b+2)(b+1) P(a-1, b+2) \\
& +k_{2}(b+1) P(a, b+1) \\
& -\left(k_{1} a+\frac{1}{2} k_{-1} b(b-1)+k_{2} b\right) P(a, b) .
\end{aligned}
$$

To apply the $\Omega$ expansion we transform $a$ to a continuous variable

$$
a=\Omega \phi_{A}+\Omega^{1 / 2} \xi .
$$

For this transformed variable set the probability density that corresponds to $P(a, b)$ is $\Pi(\xi, b)$. The left-hand side of the master equation becomes

$$
\frac{d P(a, b)}{d t} \rightarrow \frac{d \Pi(\xi, b)}{\partial t}-\Omega^{1 / 2} \frac{d \phi_{A}}{d t} \frac{\partial \Pi(\xi, b)}{\partial \xi} .
$$

Applying a Taylor series expansion in powers of $\Omega^{1 / 2}$ to Eq. (40) gives

$$
\begin{aligned}
\frac{\partial \Pi(\xi, b)}{\partial t}-\Omega^{1 / 2} \frac{d \phi_{A}}{d t} \frac{\partial \Pi(\xi, b)}{\partial \xi}= & k_{1}\left[1+\Omega^{-1 / 2} \frac{\partial}{\partial \xi}+\frac{\Omega^{-1}}{2} \frac{\partial^{2}}{\partial \xi^{2}}+\cdots\right]\left(\Omega \phi_{A}+\Omega^{1 / 2} \xi\right) \Pi(\xi, b-2) \\
& +\frac{k_{-1}}{2 \Omega}(b+2)(b+1)\left[1-\Omega^{-1 / 2} \frac{\partial}{\partial \xi}+\frac{\Omega^{-1}}{2} \frac{\partial^{2}}{\partial \xi^{2}}+\cdots\right] \Pi(\xi, b+2)+k_{2}(b+1) \Pi(\xi, b+1) \\
& -\left(k_{1}\left(\Omega \phi_{A}+\Omega^{1 / 2} \xi\right)+\frac{k_{-1}}{2 \Omega} b(b-1)+k_{2} b\right) \Pi(\xi, b),
\end{aligned}
$$


where the operator in the square brackets is applied to all of the terms on the right-hand side of the brackets. Since the consumption rate of $\mathrm{B}$ is large, the parameter $\epsilon=1 /\left(k_{-1}\right.$ $\left.+k_{2}\right)$ is small. As we have previously applied SPA, we write $\Pi(\xi, b)$ as a power series in $\epsilon$.

$$
\Pi(\xi, b)=W_{0}(\xi, b)+\epsilon W_{1}(\xi, b)+\epsilon^{2} W_{2}(\xi, b)+\cdots
$$

We now assume that $\epsilon$ and $1 / \Omega$ have similar orders. We define another parameter of $\mathcal{O}(1), \gamma$ as the product of $\epsilon$ and $\Omega$

$$
\gamma=\epsilon \Omega \text {. }
$$

Substituting the power series expansion of $\Pi$, multiplying both sides by $\epsilon$, and changing all instances of $\epsilon$ to $\gamma / \Omega$ we obtain the following expanded master equation:

$$
\begin{aligned}
\gamma \Omega^{-1} & \left(\frac{d W_{0}}{d t}+\gamma \Omega^{-1} \frac{d W_{1}}{d t}+\cdots\right)-\gamma \Omega^{-1 / 2} \frac{d \phi_{A}}{d t} \frac{\partial\left(W_{0}+\gamma \Omega^{-1} W_{1}+\cdots\right)}{\partial \xi}=k_{1}\left[1+\Omega^{-1 / 2} \frac{\partial}{\partial \xi}+\frac{\Omega^{-1}}{2} \frac{\partial^{2}}{\partial \xi^{2}}+\cdots\right]\left(\gamma \phi_{A}+\gamma \Omega^{-1 / 2} \xi\right) \\
& \times\left(W_{0}(\xi, b-2)+\gamma \Omega^{-1} W_{1}(\xi, b-2)+\cdots\right)+\frac{K_{-1}}{2}(b+2)(b+1)\left[\Omega^{-1}-\Omega^{-3 / 2} \frac{\partial}{\partial \xi}+\frac{\Omega^{-2}}{2} \frac{\partial^{2}}{\partial \xi^{2}}+\cdots\right]\left(W_{0}(\xi, b+2)\right. \\
& \left.+\gamma \Omega^{-1} W_{1}(\xi, b+2)+\cdots\right)+K_{2}(b+1)\left(W_{0}(\xi, b+1)+\gamma \Omega^{-1} W_{1}(\xi, b+1)+\cdots\right) \\
& -\left(k_{1}\left(\gamma \phi_{A}+\gamma \Omega^{-1 / 2} \xi\right)+\frac{K_{-1} \Omega^{-1}}{2} b(b-1)+K_{2} b\right)\left(W_{0}(\xi, b)+\gamma \Omega^{-1} W_{1}(\xi, b)+\cdots\right) .
\end{aligned}
$$

\section{A. $\Omega^{0}$ terms}

Collection the highest-order terms of Eq. (42) results in

$$
\begin{aligned}
0= & k_{1} \gamma \phi_{A} W_{0}(\xi, b-2)+K_{2}(b+1) W_{0}(\xi, b+1) \\
& -\left(k_{1} \gamma \phi_{A}+K_{2} b\right) W_{0}(\xi, b) .
\end{aligned}
$$

Since none of the expressions that multiply the joint density terms depend on $\xi$, integrating Eq. (43) over all values of $\xi$ changes the joint densities to marginal densities.

$$
\begin{aligned}
0= & k_{1} \gamma \phi_{A} W_{0}(b-2)+K_{2}(b+1) W_{0}(b+1) \\
& -\left(k_{1} \gamma \phi_{A}+K_{2} b\right) W_{0}(b) .
\end{aligned}
$$

The analytical expression for $W_{0}(b)$ is

$$
W_{0}(b)=\exp \left(-\frac{3}{2 \kappa}\right) \sum_{n=\operatorname{ceil}(b / 2)}^{b}\left(\frac{1}{2}\right)^{b-n}\left(\frac{\kappa^{-n}}{n !}\right)\left(\begin{array}{c}
n \\
b-n
\end{array}\right),
$$

where $\kappa=K_{2} /\left(k_{1} \gamma \phi_{A}\right)$. If the first terms of Eq. (43) contained a $W_{0}(b-1)$ term instead of $W_{0}(b-2)$, then $W_{0}(b)$ would follow a simple Poisson distribution. Finding an expression for $W_{0}(b)$ for an arbitrary reaction mechanism may not be a trivial task. Evaluating such a probability density, calculating moments, or expected values of functions sampling this density may be computationally intensive tasks as well. Such challenges are not the focus of this paper, however. What we have learned from the $\Omega^{0}$ terms of the perturbation analysis is that the density of $b$ is uncoupled from the fluctuations of the non-QSSA species at zero order, and, therefore, $W_{0}(\xi, b)=W_{0}(\xi) W_{0}(b)$.
Figure 12 shows the probability densities of $b$ that correspond to times 0.5 and $1.5 \mathrm{~s}$ of the SSA simulations shown in Fig. 11. The full model probability densities were calculated from 25000 SSA simulations. The sQSPA- $\Omega$ probability density was calculated from the perturbation analysis estimate [Eq. (45)]. This figure shows that the sQSPA- $\Omega$ model approaches the full model in the limit of large $\Omega$ and fast consumption of $b$.

\section{B. $\Omega^{-1 / 2}$ terms}

Collecting the terms of Eq. (42) with $\Omega^{-1 / 2}$ terms gives

$$
\begin{aligned}
-\gamma \frac{d \phi_{A}}{d t} \frac{\partial W_{0}(\xi, b)}{\partial \xi}= & k_{1} \frac{\partial}{\partial \xi}\left(\gamma \phi_{A} W_{0}(\xi, b-2)\right) \\
& +k_{1} \gamma \xi W_{0}(\xi, b-2)-k_{1} \gamma \xi W_{0}(\xi, b) .
\end{aligned}
$$

Summing Eq. (46) over all values of $b$ gives the following deterministic evolution equation:

$$
\frac{d \phi_{A}}{d t}=-k_{1} \phi_{A}
$$

This is the same evolution equation that arises from applying dQSPA to this reaction mechanism. However, it is different than the sQSPA model.

Using the property of the statistical independence of $\xi$ and $b$, Eq. (46) can be manipulated to show that $W_{0}(\xi)$ is a normal distribution with zero mean and a standard deviation of $\sqrt{\phi_{A}}$. Although it did not occur in this example, situations 


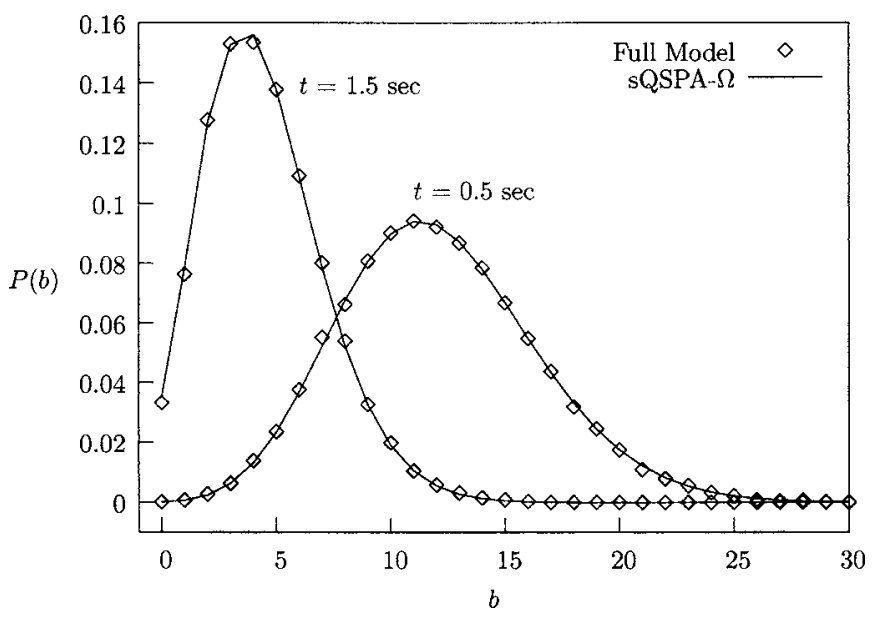

FIG. 12. The probability density of $b$, reacting according to mechanism (38) and the parameters listed in this section. The full model estimate was calculated from 25000 SSA simulations. The sQSPA- $\Omega$ estimate was calculated using Eq. (45).

do arise in which the deterministic evolution equation for the non-QSSA species concentrations depend upon the solution to $W_{0}(b)$.

\section{C. $\Omega^{-1}$ terms}

Collecting the terms of Eq. (42) with $\Omega^{-1}$ and summing over all values of $b$ gives a linear Fokker-Planck equation for the evolution of $\xi$.

$$
\frac{d W_{0}(\xi)}{d t}=k_{1} \gamma \frac{\partial\left(\xi W_{0}(\xi)\right)}{\partial \xi}+\frac{k_{1} \gamma \phi_{A}}{2} \frac{\partial^{2} W_{0}(\xi)}{\partial \xi^{2}}
$$

The $\mathrm{sQSPA}-\Omega$ procedure just described is useful when non-QSSA species are evolving deterministically or experience small Gaussian fluctuations about the deterministic solution, and the QSSA species are small. This model reduction gives a discrete probability density for the QSSA species and a deterministic value for the non-QSSA species. It also reduces the number of large parameters in the system. In the example above there are three kinetic parameters and one size parameter that can vary over orders of magnitude and are difficult to estimate from slow time scale measurements. In the SQSPA- $\Omega$ one parameter $\left(k_{1}\right)$ of $\mathcal{O}(1)$ is needed to solve deterministic evolution equations for $\phi_{A}$. Two parameters $\left(k_{1}, \gamma\right)$ of $\mathcal{O}(1)$ are needed to solve the Fokker-Planck equation for $\xi$, and three parameters $\left(k_{1}, \gamma, K_{2}\right)$ of $\mathcal{O}(1)$ are needed to solve for the probability density of $b$.

\section{CONCLUSIONS}

In this work, we have developed two methods for eliminating QSSA species from the chemical master equation when the populations for these species are small enough to require a stochastic description. Both model reductions use singular perturbation analysis and therefore in the limit of a parameter approaching zero, the solution of the reduced master equation converges to the solution of the full model's master equation.

The first model reduction is applicable when the QSSA species numbers usually sample zero, and is called sQSPA.
The reduced master equation from this order reduction gives a new reaction mechanism and corresponding reaction rate expressions that apply on the slow time scale. The new mechanism can be simulated with Gillespie's SSA. We have shown how this model reduction can be applied to a generic reaction network.

In some cases the reduced mechanism and reduced reaction rates from SQSPA are identical to those determined from the dQC models. This is often not the case, however. Therefore, we recommend against using reduced reaction mechanisms and their corresponding reaction rates derived from classical deterministic QSSA in stochastic simulations. We have shown theoretically and numerically that application of dQC mechanisms does not reproduce the probability density of the non-QSSA species of the full model. We recommend in stochastic simulations that highly reactive species be removed from the mechanism using the sQSPA model presented in this paper. We have also shown numerically that application of sQSPA mechanisms does reproduce the probability density of the non-QSSA species of the full model. We also recommend against using reduced mechanisms and rate expressions derived from sQSPA or sQSPA- $\Omega$ in differential equation models.

The second model reduction is applicable when the nonQSSA species are large enough to merit a deterministic description, while the QSSA species numbers are small and stochastic. This model reduction is performed using SPA in conjunction with an $\Omega$ expansion.

The benefits of both the stochastic QSSA reduced models developed here are similar to the benefits of QSSA in deterministic models: smaller models with faster simulation times are created, and fast time-scale kinetic parameters are eliminated.

\section{ACKNOWLEDGMENTS}

Funding was provided by NIH grant 5R21AI071197-02 and NSF DDDAS grant CNS-0540147. One of the authors (E.L.H.) gratefully acknowledges support from the National Institutes of Health under Ruth L. Kirschstein National Research Service Award 5F32CA120055. The authors would like to thank Dr. J. W. Eaton for help preparing this paper. All simulations were performed using Octave (http:// www.octave.org). Octave is freely distributed under the terms of the GNU General Public License.

${ }^{1}$ D. T. Gillespie, J. Phys. Chem. 81, 2340 (1977).

${ }^{2}$ D. T. Gillespie, Physica A 188, 404 (1992).

${ }^{3}$ E. L. Haseltine and J. B. Rawlings, J. Chem. Phys. 123, 164115 (2005).

${ }^{4}$ Y. Cao, D. T. Gillespie, and L. R. Petzold, J. Chem. Phys. 122, 014116 (2005).

5 J. Goutsias, J. Chem. Phys. 122, 184102 (2005).

${ }^{6}$ A. Samant and D. G. Vlachos, J. Chem. Phys. 123, 144114 (2005).

${ }^{7}$ H. Salis and Y. Kaznessis, J. Chem. Phys. 122, 054103 (2005).

${ }^{8}$ D. T. Gillespie, J. Chem. Phys. 115, 1716 (2001).

${ }^{9}$ E. L. Haseltine and J. B. Rawlings, J. Chem. Phys. 117, 6959 (2002); URL: http://ojps.aip.org/jcpo/?jsessionid=2327091025045033306

${ }^{10}$ D. T. Gillespie and L. R. Petzold, J. Chem. Phys. 119, 8229 (2003).

${ }^{11}$ M. Rathinam, L. R. Petzold, Y. Cao, and D. T. Gillespie, J. Chem. Phys. 119, 12784 (2003).

${ }^{12}$ T. Tian and K. Burrage, J. Chem. Phys. 121, 10356 (2004).

${ }^{13}$ A. Chatterjee, D. G. Vlachos, and M. A. Katsoulakis, J. Chem. Phys. 122, $024112(2005)$. 
${ }^{14}$ J. B. Rawlings and J. G. Ekerdt, Chemical Reactor Analysis and Design Fundamentals (Nob Hill, Madison, WI, 2002).

${ }^{15}$ T. Turanyi, A. Tomlin, and M. Pilling, J. Phys. Chem. 97, 163 (1993).

${ }^{16}$ W. M. Deen, Analysis of Transport Phenomena, Topics in Chemical Engineering (Oxford University Press, New York, 1998).

${ }^{17}$ W. Klonowski, Biophys. Chem. 18, 73 (1983).

${ }^{18}$ C. V. Rao and A. P. Arkin, J. Chem. Phys. 118, 4999 (2003).

${ }^{19}$ J. A. M. Janssen, J. Stat. Phys. 57, 171 (1989).

${ }^{20}$ J. A. M. Janssen, J. Stat. Phys. 57, 187 (1989).

${ }^{21}$ N. G. van Kampen, Phys. Rep. 124, 69 (1985).

${ }^{22}$ S. Peles, B. Munsky, and M. Khammash, J. Chem. Phys. 125, 204104 (2006).
${ }^{23}$ M. V. Henri, in Foundation Stones of Biochemistry, edited by T. R. C. Boyde (Voile et Aviron, Hong Kong, 1980) (English translation).

${ }^{24}$ L. Michaelis and M. L. Menten, Biochem. Z. 49, 333 (1913).

${ }^{25}$ L. Michaelis and M. L. Menten, in Foundation Stones of Biochemistry, edited by T. R. C. Boyde (Ref. 23) (English translation).

${ }^{26}$ G. E. Briggs and J. B. S. Haldane, Biochem. J. 19, 338 (1925).

${ }^{27}$ F. Heineken, H. Tsuchiya, and R. Aris, Math. Biosci. 1, 95 (1967).

${ }^{28}$ S. Schnell and P. K. Maini, Math. Comput. Modell. 35, 137 (2001).

${ }^{29}$ L. A. Segel and M. Slemrod, SIAM Rev. 31, 446 (1989).

${ }^{30}$ N. G. van Kampen, Stochastic Processes in Physics and Chemistry, 2nd ed. (Elsevier Science Amsterdam, The Netherlands, 1992). 